\title{
CONTRIBUTIONS TO THE THEORY OF SURFACES IN A 4-SPACE OF CONSTANT CURVATURE
}

\author{
BY \\ YUNG-CHOW WONG(')
}

1.1. Introduction. A Riemannian 4-space is a space of constant curvature and is denoted by $S_{4}$, if its Riemann tensor is of the form

$$
R_{k \lambda \mu}^{\rho}=-C\left(a_{k \lambda} \delta_{\mu}^{\rho}-a_{\kappa \mu} \delta_{\lambda}^{\rho}\right) \quad(\rho, \kappa, \lambda, \mu=1, \cdots, 4),
$$

where $a_{k \lambda}$ is the fundamental tensor, $\delta_{\mu}^{\rho}$ the Kronecker delta and $C$ a scalar, which is automatically a constant. A Euclidean 4-space, which we denote by $R_{4}$, is a special case of $S_{4}$. In this paper we study a few special surfaces $V_{2}$ in an $S_{4}$, including those in an $R_{4}$. The method used is invariant and is similar to those of Ricci( $\left.{ }^{2}\right)$ [14] and Graustein [9] for their studies of surfaces in a Euclidean 3-space. In essence, we first set up a suitable system of invariant fundamental equations for a $V_{2}$ in $S_{4}$ and then express the imbedding requirements of $V_{2}$ in $S_{4}$ in terms of the intrinsic properties of $V_{2}$. $\$ 1.2$ contains some formulas in the intrinsic theory of $V_{2}$ which are useful for our later work. In $\$ 2$, the curvature tensor $H_{c b}^{\cdot{ }^{k}}$ and the curvature conic $(G)$ for a $V_{2}$ in $S_{4}$ are introduced and discussed. Regarding $H_{c b}^{\cdot{ }^{*}}$ as a pencil of tensors, we find a certain relationship between the nature of the pencil and that of the conic $(G)$. Some formulas concerning $(G)$ are then given, including one which leads to an interesting interpretation of the Gaussian curvature of $V_{2}$ in terms of $(G) . \S 3$ contains the fundamental equations for $V_{2}$ in $S_{4}$ in invariant form and an outline of the method used for the study of some surfaces with preassigned curvature properties. $\$ 4$ is devoted to the study of minimal $V_{2}$, and, in particular, the $R$-surface of Kommerell. In $\S 5$, ruled surfaces in $S_{4}$ are considered. $\S 6$ gives a special imbedding of $R_{2}$ in $R_{4}$. The paper ends in $\$ 7$ with the determination of those $V_{2}$ in $S_{4}$ whose first fundamental form and one of whose second fundamental forms are respectively identical with the first and second fundamental forms of a $V_{2}$ in $S_{3}$.

1.2. Some useful formulas. Here are some intrinsic formulas for a $V_{2}$, which will be used in our later work and can be verified readily. Let $g_{c b}$ $(a, b, c=1,2)$ be the fundamental tensor of a $V_{2}$ which is supposed to be positive definite, and let $i_{(1)}^{a}, i_{(2)}^{a}$ be two mutually orthogonal congruences (that

Presented to the Society April 27, 1946; received by the editors October 10, 1945.

(1) Most of the results in this paper were obtained while the author was a Harrison Research Fellow at the University of Pennsylvania. The author wishes to thank Professor D. J. Struik of the Massachusetts Institute of Technology for his encouragement which led to the writing of this paper.

(2) Numbers in brackets refer to the literature listed at the end of the paper. 
is, unit vector fields) in $V_{2}$. Then

$$
g_{c b}=i_{c}^{(1)} i_{b}^{(1)}+i_{c}^{(2)} i_{b}^{(2)}, \quad i_{(b)}^{a} i_{a}^{(c)}=\delta_{b}^{c}, \quad i_{b}^{(a)} i_{(a)}^{c}=\delta_{b}^{b}
$$

where $i_{a}^{(b)}=g_{a c} i_{(b)}^{c}$, and $\delta_{b}^{c}$ is the Kronecker delta.

The geodesic curvatures (to within sign) of the two congruences $i_{(1)}^{a}, i_{(2)}^{a}$ are, respectively,

$$
\beta=-i_{(2)}^{b} i_{(1)}^{c} i_{b, c}^{(1)}, \quad \alpha=-i_{(1)}^{b} i_{(2)}^{c} i_{b, c}^{(2)}
$$

where the comma denotes covariant differentiation with respect to $g_{c b}$.

Let us write

$$
\delta_{1} f=i_{(1)}^{c} f_{, c}, \quad \delta_{2} f=i_{(2)}^{c} f_{, c}
$$

as the directional derivatives along the congruences $i_{(1)}^{a}, i_{(2)}^{a}$. Then the integrability condition for $\delta_{1} f, \delta_{2} f$ is

$$
\left(\delta_{2} \delta_{1}-\delta_{1} \delta_{2}+\beta \delta_{1}-\alpha \delta_{2}\right) f=0,
$$

that is, (1.4) is the necessary and sufficient condition that two scalars, which we denote by $\delta_{1} f, \delta_{2} f$, be the directional derivatives along $i_{(1)}^{a}, i_{(2)}^{a}$.

The first and second differential parameters for $g_{c b}$ are, respectively,

$$
\Delta_{1} f=\left(\delta_{1} f\right)^{2}+\left(\delta_{2} f\right)^{2}, \quad \Delta_{2} f=\left(\delta_{1} \delta_{1}+\delta_{2} \delta_{2}+\alpha \delta_{1}+\beta \delta_{2}\right) f .
$$

The Gaussian curvature $K$ (that is, the scalar curvature) of $V_{2}$ is given by the Liouville formula:

$$
K=-\left(\delta_{1} \alpha+\delta_{2} \beta+\alpha^{2}+\beta^{2}\right) .
$$

(1.5) and (1.6) are of course independent of the choice of $i_{(1)}^{a}, i_{(2)}^{a}$.

The condition for $i_{(1)}^{a}, i_{(2)}^{a}$ to be an isometric net is

$$
\delta_{2} \alpha-\delta_{1} \beta=0 \text {. }
$$

If $\bar{i}_{(1)}^{a}, \bar{i}_{(2)}^{a}$ are two other mutually orthogonal congruences defined by

$$
\bar{i}_{(1)}^{a}=i_{(1)}^{a} \cos \theta+i_{(2)}^{a} \sin \theta, \quad \epsilon_{(2)}^{a}=-i_{(1)}^{a} \sin \theta+i_{(2)}^{a} \cos \theta(\epsilon= \pm 1),
$$

then

$$
\begin{gathered}
\bar{\delta}_{1}=\cos \theta \delta_{1}+\sin \theta \delta_{2}, \quad \bar{\epsilon}_{2}=-\sin \theta \delta_{1}+\cos \theta \delta_{2} ; \\
\bar{\alpha}=-\left(\delta_{1} \theta-\beta\right) \sin \theta+\left(\delta_{2} \theta+\alpha\right) \cos \theta, \\
-\epsilon \bar{\beta}=\left(\delta_{1} \theta-\beta\right) \cos \theta+\left(\delta_{2} \theta+\alpha\right) \sin \theta .
\end{gathered}
$$

From (1.9) and (1.10) we have

$$
\epsilon\left(\bar{\delta}_{2} \bar{\alpha}-\bar{\delta}_{1} \bar{\beta}\right)=\delta_{2} \alpha-\delta_{1} \beta+\Delta_{2} \theta .
$$

Therefore, if $i_{(1)}^{a}, i_{(2)}^{a}$ are an isometric net, then $\bar{i}_{(1)}^{a}, i_{(2)}^{a}$ are also one if and only if $\Delta_{2} \theta=0$. 
For the linear element (that is, fundamental form)

$$
d s^{2}=E d u^{2}+G d v^{2},
$$

we have

$$
\delta_{1}=\frac{\partial_{u}}{E^{1 / 2}}, \quad \delta_{2}=\frac{\partial_{v}}{G^{1 / 2}} \quad\left(\partial_{u} \equiv \frac{\partial}{\partial u}, \partial_{v} \equiv \frac{\partial}{\partial v}\right),
$$

$$
\begin{aligned}
& \alpha=\frac{1}{E^{1 / 2}} \partial_{u} \log G^{1 / 2}, \quad \beta=\frac{1}{G^{1 / 2}} \partial_{v} \log E^{1 / 2} \\
& K=-\frac{1}{(E G)^{1 / 2}}\left[\partial_{u}\left(\frac{\partial_{u} G^{1 / 2}}{E^{1 / 2}}\right)+\partial_{v}\left(\frac{\partial_{v} E^{1 / 2}}{G^{1 / 2}}\right)\right]
\end{aligned}
$$

For the linear element

$$
d s^{2}=2 F d u d v
$$

we have

$$
\Delta_{2}=\frac{2}{F} \partial_{v} \partial_{u}, \quad K=-\frac{1}{F} \partial_{v} \partial_{u} \log F .
$$

Closely connected with (1.14) is the Liouville equation (cf. Knoblauch [10, p. 543])

$$
\partial_{\nu} \partial_{u} \log \lambda=A \lambda^{B} \quad(A, B \text { constants), }
$$

the solution of which is

$$
\lambda^{B}=-\frac{2 U^{\prime} V^{\prime}}{A B(1+U V)^{2}},
$$

where $U, V$ are respectively arbitrary non-constant functions of $u, v$ alone, and the prime denotes differentiation.

2.1. The curvature tensor $H_{i c}^{\cdot k}$. We shall use the general notation of Schouten-Struik $[15$, chaps. 2,3$]$. Let $y^{\kappa}(\kappa, \lambda, \cdots=1, \cdots, 4)$ be the coordinates in $S_{4}\left({ }^{(}\right)$, with positive definite fundamental tensor $a_{\lambda_{k}}$, and let $\Gamma_{\lambda_{k}}^{\mu}$, $\nabla_{\mu}$ be the Christoffel symbol of the second kind and the symbol of covariant differentiation with respect to $a_{\lambda_{k}}$, respectively.

In $S_{4}$ we introduce a surface $V_{2}$ by the equations

$$
y^{k}=y^{k}\left(u^{a}\right)
$$$$
(a, b=1,2) \text {. }
$$

Then the first fundamental tensor induced in $V_{2}$ by $S_{4}$ is

$$
g_{c b}=a_{\lambda x} B_{c}^{\lambda} B_{b}^{k},
$$

(8) Although the definitions and results in $\$ \$ 2.1-2.3$ are stated for a $V_{2}$ in an $S_{4}$, most of them also hold for a $V_{2}$ in any Riemannian 4-space. 
where

$$
B_{b}^{*}=\partial_{b} y^{*} .
$$

At each point $P$ of $V_{.2}$, the connecting tensor $B_{b}^{k}$ spans the tangent plane to $V_{2}$ at $P$. Let $i_{(p)}^{*}(p, q=3,4)$ be two mutually orthogonal unit vectors normal to $V_{2}$, defined at each point of $V_{2}$. Then

$$
h_{c b}^{(p)}=-B_{c}^{\lambda} B_{b}^{k} \nabla_{\lambda} i_{k}^{(p)},
$$

where $i_{k}^{(p)}=a_{k \lambda} i_{(p)}^{\lambda}$, is the second fundamental tensor of $V_{2}$ in $S_{4}$ for the normal $i_{(p)}^{\kappa}$. The curvature tensor

$$
H_{c b}^{\cdot \cdot k}=h_{c b}^{(3)} \ddot{i}_{(8)}^{k}+h_{c b}^{(4)} i_{(4)}^{k}
$$

is independent of the choice of $i_{(p)}^{\kappa}$.

Two directions $i^{a}, j^{a}$ in $V_{2}$ are called conjugate if

$$
H_{c b}^{\cdot *{ }^{*}} i^{c} j^{b}=0 \text {. }
$$

In particular, if a direction $j^{a}$ satisfies

$$
H_{c b}^{\cdot *} j^{c} j^{b}=0 \text {, }
$$

it is called an asymptotic direction. In general, there are two and only two conjugate directions but no asymptotic direction.

Let $(C)$ be a curve on $V_{2}$ passing through $P$ and with unit tangent vector $i^{a}$ at $P$. Then the component

$$
\overline{P Q}=u^{\star}
$$

in the normal plane of $V_{2}$ of the curvature vector of $(C)$ with respect to $S_{4}$ is

$$
u^{k}=H_{c b}^{*{ }^{k}} i^{c} i^{b},
$$

which depends only on $i^{a}$ and is called the normal curvature vector of $V_{2}$ in $S_{4}$ for the direction $i^{a}$ at $P$. The locus $(G)$ of the end point $Q$ as $i^{a}$ takes all the possible positions in the tangent plane is an ellipse, proper or degenerate, in the normal plane. We shall call $(G)$ the curvature conic of $V_{2}$ in $S_{4}$ at $P$.

From (2.5) it follows that the equations of $(G)$ in the rectangular Cartesian coordinates $z, t$ with axes $i_{(3)}^{\kappa}, i_{(4)}^{\kappa}$ are

$$
z=h_{c b}^{(3)} i^{c} i^{b}, \quad t=h_{c b}^{(4)} i^{c} i^{b} .
$$

2.2. The tensor $H_{\ddot{c}{ }^{*}}$ and the conic $(G)$. Owing to the arbitrary choice of $i_{(3)}^{\kappa}$ in (2.5), $H_{\ddot{c}}^{\ddot{k}^{k}}$ may be considered as a pencil of tensors $\lambda h_{c b}^{(3)}+\mu h_{c b}^{(4)}$. We shall now proceed to prove the following relationship between the characteristics of this pencil (in the sense of the theory of elementary divisors $\left({ }^{4}\right)$ ) and

( $\left.{ }^{(}\right)$See, for example, Bôcher [1, chaps. 20-21]. 
the geometric properties of $(G)$ and $P$.

THEOREM 2.1. If the pencil $H_{\ddot{c}{ }^{*}}$ is not singular, we denote as usual the characteristics of the pencil by squared brackets. Then for:

[11], $(G)$ is an ellipse not passing through $P$, which degenerates into a line segment if and only if the conjugate directions of $V_{2}$ are orthogonal to each other.

[(11)], $(G)$ is a line segment passing through $P$ but not ending at $P$.

[2], $(G)$ is a non-degenerate ellipse passing through $P$.

If the pencil of tensors is singular, $(G)$ is a line segment ending at $P$.

Proof. We first suppose that there is a nonsingular tensor in the pencil. Let it be $h_{c b}^{(3)}$. Then we have the following cases.

Case 1. [11]. A coordinate system( $\left(^{5}\right) u^{a}$ in $V_{2}$ exists so that at the point $P$ under consideration the equations (2.9) of $(G)$ take the form

$$
z=\epsilon_{1}\left(i^{1}\right)^{2}+\epsilon_{2}\left(i^{2}\right)^{2}, \quad t=\sigma_{1}\left(i^{1}\right)^{2}+\sigma_{2}\left(i^{2}\right)^{2},
$$

where $\epsilon_{1}, \epsilon_{2}= \pm 1 ; \sigma_{1}, \sigma_{2}$ are scalars, and $i^{a}$ is a variable unit vector, that is, its components $i^{1}, i^{2}$ are subject only to the condition

$$
g_{c b} i^{c} i^{b}=1 \text {. }
$$

The conjugate directions of $V_{2}$ at $P$ are evidently

$$
\dot{j}_{(1)}^{a}=(1,0), \quad \dot{j}_{(2)}^{a}=(0 ; 1) .
$$

From (2.10) and (2.11) it follows that $(G)$ is an ellipse. If $(G)$ passes through $P$, the equations $z=t=0$ are satisfied by some $i^{a}$. This requires that $\sigma_{1} / \epsilon_{1}=\sigma_{2} / \epsilon_{2} \equiv \sigma$. Then the subcase [(11)] arises, and (2.10) become

$$
z=\epsilon_{1}\left(i^{1}\right)^{2}+\epsilon_{2}\left(i^{2}\right)^{2}, . \quad t=\sigma z,
$$

showing that $(G)$ is a line segment not ending at $P$.

Let us now return to the general case $(2.10)$. The condition for $(G)$ to be a line segment not passing through $P$ is that $\lambda$, $\mu$ exist such that $\lambda z+\mu t=1$, that is, by (2.10). that

$$
\left(\lambda \epsilon_{1}+\mu \sigma_{1}\right)\left(i^{1}\right)^{2}+\left(\lambda \epsilon_{2}+\mu \sigma_{2}\right)\left(i^{2}\right)^{2}=1
$$

should be true for some $i^{1}, i^{2}$ satisfying (2.11). Subtraction of the above equation and (2.11) gives

$$
\left(\lambda \epsilon_{1}+\mu \sigma_{1}-g_{11}\right)\left(i^{1}\right)^{2}-2 g_{12} i^{1} i^{2}+\left(\lambda \epsilon_{2}+\mu \sigma_{2}-g_{22}\right)\left(i^{2}\right)^{2}=0 .
$$

Since this should be true for all values of $i^{1}, i^{2}$, we have

$$
\begin{gathered}
g_{12}=0, \\
\lambda \epsilon_{1}+\mu \sigma_{1}=g_{11}, \quad \lambda \epsilon_{2}+\mu \sigma_{2}=g_{22} .
\end{gathered}
$$

(b) We note that $u^{a}$ are either real or complex conjugate. If they are complex conjugate, $\epsilon_{1}=\epsilon_{2} ;$ and $\sigma_{1}, \sigma_{2} ; i^{1}, i^{2}$ are complex conjugate, $z, t$ being always real. 
Equation (2.14) shows that the conjugate directions (2.12) are orthogonal to each other. If $\sigma_{1} / \epsilon_{1} \neq \sigma_{2} / \epsilon_{2}$, the two equations (2.15) have a unique solution for $\lambda, \mu$; for, this is not true only when $g_{11}=g_{22}=0$, but then we have, because of (2.14), $g_{c b}=0$. If $\sigma_{1} / \epsilon_{1}=\sigma_{2} / \epsilon_{2} \equiv \sigma$, then $z=\sigma t$, and we have a line segment passing through $P$.

We have now proved our assertions in Theorem 2.1 for the subcases [11] and [(11)]. Incidentally we remark that for the subcase [11] the two tangents from $P$ to $(G)$ are the normal curvature vectors for the conjugate directions (2.12). In fact, the line $\lambda z+\mu t=0$ cuts $(G)$ in two points for which

$$
\left(\lambda \epsilon_{1}+\mu \sigma_{1}\right)\left(i^{1}\right)^{2}+\left(\lambda \epsilon_{2}+\mu \sigma_{2}\right)\left(i^{2}\right)^{2}=0,
$$

and therefore if the two points coincide, they do so either at $i^{1}=0$, or at $i^{2}=0$.

Case 2. [2]. A (real) coordinate system $u^{a}$ in $V_{2}$ exists such that at $P$ the equations (2.9) of $(G)$ take the form

$$
z=2 i^{1} i^{2}, \quad t=2 \sigma_{3} i^{1} i^{2}+\sigma_{2}\left(i^{2}\right)^{2} \quad\left(\sigma_{2} \neq 0\right) .
$$

The $i^{1}, i^{2}$ satisfy (2.11), where $g_{11} \neq 0$ since $g_{c b}$ is positive definite and the coordinates $u^{a}$ are real. The $(G)$ as given by $(2.16)$ is an ellipse passing through $P$; for, $i^{2}=0$ is part of a solution of the equations $z=t=0$ and (2.11). The point $P$ on $(G)$ corresponds to the asymptotic direction $j_{(1)}^{a}=(1,0)$. If $(G)$ were a line segment, we should have $t / z=\sigma_{3}+\sigma_{2} i^{2} / i^{1}=$ const. But this is impossible since $\sigma_{2} \neq 0$. Hence $(G)$ is a proper ellipse.

If all the tensors in the pencil $H_{\ddot{c}{ }^{*}}$ are singular, there exists a (real) coordinate system $u^{a}$ in $V_{2}$ such that the equations (2.9) of $(G)$ take the form

$$
z=0, \quad t=\sigma_{1}\left(i^{1}\right)^{2} .
$$

The conic $(G)$ is therefore a line segment ending at $P$.

2.3. Some formulas concerning $(G)$. Let all the tensors in $V_{2}$ be expressed in terms of two mutually orthogonal unit vectors $i_{(1)}^{a}, i_{(2)}^{a}$ and some scalars. Thus,

$$
\begin{aligned}
h_{c b}^{(3)} & =\rho_{1} i_{c}^{(1)} i_{b}^{(1)}+\rho_{2} i_{c}^{(2)} i_{b}^{(2)}+\rho_{3}\left(i_{c}^{(1)} i_{b}^{(2)}+i_{c}^{(2)} i_{b}^{(1)}\right), \\
h_{c b}^{(4)} & =\sigma_{1} i_{c}^{(1)} i_{b}^{(1)}+\sigma_{2} i_{c}^{(2)} i_{b}^{(2)}+\sigma_{3}\left(i_{c}^{(1)} i_{b}^{(2)}+i_{c}^{(2)} i_{b}^{(1)}\right), \\
i^{a} & =i_{(1)}^{a} \cos \phi+i_{(2)}^{a} \sin \phi .
\end{aligned}
$$

The equations of the curvature conic $(G)$ are therefore, by (2.19),

$$
\begin{aligned}
z & =2^{-1}\left(\rho_{1}+\rho_{2}\right)+2^{-1}\left(\rho_{1}-\rho_{2}\right) \cos 2 \phi+\rho_{3} \sin 2 \phi, \\
t & =2^{-1}\left(\sigma_{1}+\sigma_{2}\right)+2^{-1}\left(\sigma_{1}-\sigma_{2}\right) \cos 2 \phi+\sigma_{3} \sin 2 \phi .
\end{aligned}
$$

Elementary calculations show that

$$
d^{2}=4^{-1}\left[\left(\rho_{1}+\rho_{2}\right)^{2}+\left(\sigma_{1}+\sigma_{2}\right)^{2}\right],
$$

where $d$ is the distance from $P$ to the center of $(G)$. 


$$
\begin{gathered}
\text { Area of }(G)= \pm 2^{-1} \pi\left[\left(\rho_{1}-\rho_{2}\right) \sigma_{3}-\left(\sigma_{1}-\sigma_{2}\right) \rho_{3}\right] \\
\qquad r^{2}=4^{-1}\left[\left(\rho_{1}-\rho_{2}\right)^{2}+\left(\sigma_{1}-\sigma_{2}\right)^{2}\right]+\rho_{3}^{2}+\sigma_{3}^{2}
\end{gathered}
$$

where $r$ is the radius of the director circle of $(G)$.

From these, it follows that the power of $P$ with respect to the director circle of $(G)$ is

$$
d^{2}-r^{2}=\rho_{1} \rho_{2}+\sigma_{1} \sigma_{2}-\stackrel{2}{\rho_{3}}-\sigma_{3}^{2} .
$$

When $V_{2}$ is in an $S_{4}$ with constant scalar curvature $C$, we see from the Gauss equation (3.6) $)_{1}$, which is the first equation in equations (3.6) of $\$ 3$, that the right-hand member of the above equation is the Gaussian curvature $K$ of $V_{2}$ minus $C$. Hence we have the following theorem.

TheOREM 2.2. At any point $P$ of $a V_{2}$ in an $S_{4}\left(\right.$ or $\left.R_{4}\right)$, the power of $P$ with respect to the director circle of $(G)$ is equal to the Gaussian curvature of $V_{2}$ minus the scalar curvature of $S_{4}$ (or to the Gaussian curvature of $V_{2}$ ). In particular, at any $P$ of $a V_{2}$ in an $R_{4}$, the Gaussian curvature of $V_{2}$ is greater than 0 , equal to 0 , or less than 0 according as $P$ lies outside, on, or inside the director circle of $(G)$.

From the equations (2.20) of $(G)$ and the Gauss equation (3.6) $)_{1}$ of $V_{2}$ in $S_{4}$, it can be shown by elementary calculation that the angle $\Omega$ subtended by $(G)$ at $P$ is given by

$$
(\tan \Omega)^{2}=\Delta /(K-C)^{2},
$$

where

$$
\Delta=\left(\sigma_{1} \rho_{2}-\sigma_{2} \rho_{1}\right)^{2}+4\left(\sigma_{3} \rho_{1}-\sigma_{1} \rho_{3}\right)\left(\sigma_{3} \rho_{2}-\sigma_{2} \rho_{3}\right) .
$$

Putting $K=C$ in (2.24) we get a partial verification of Theorem 2.2. A further consequence of (2.24) is that $(G)$ passes through $P$ if and only if $\Delta=0$.

Another interesting formula concerning $(G)$ is the one that gives the angle $\omega$ between the two conjugate directions of $V_{2}$ in $S_{4}$ :

$$
(\tan \omega)^{2}=\Delta \pi^{2} / 4(\text { Area of }(G))^{2} .
$$

This can be proved as follows. By definition (2.6), the conjugate directions $i^{a}, j^{a}$ are given by

$$
h_{c b}^{(3)} i^{c} j^{b}=0, \quad h_{c b}^{(4)} i j^{c}=0,
$$

and hence by

$$
\left|\begin{array}{l}
h_{c b}^{(3)} i^{c} \\
h_{c b}^{(4)} i^{c}
\end{array}\right|=0 .
$$

But from (2.18) and (2.19), we have 


$$
\begin{aligned}
& h_{c b}^{(3)} i^{c}=\left(\rho_{1} \cos \phi+\rho_{3} \sin \phi\right) i_{b}^{(1)}+\left(\rho_{2} \sin \phi+\rho_{3} \cos \phi\right) i_{b}^{(2)}, \\
& h_{c b}^{(4)} i^{c}=\left(\sigma_{1} \cos \phi+\sigma_{3} \sin \phi\right) i_{b}^{(1)}+\left(\sigma_{2} \sin \phi+\sigma_{3} \cos \phi\right) i_{b}^{(2)} .
\end{aligned}
$$

Therefore (2.28) is equivalent to

$$
\left|\begin{array}{c}
\rho_{1} \cos \phi+\rho_{3} \sin \phi, \rho_{2} \cos \phi+\rho_{3} \sin \phi \\
\sigma_{1} \cos \phi+\sigma_{3} \sin \phi, \sigma_{2} \cos \phi+\sigma_{3} \sin \phi
\end{array}\right|=0,
$$

that is

$$
\left|\begin{array}{l}
\rho_{1} \rho_{3} \\
\sigma_{1} \sigma_{3}
\end{array}\right| \cos ^{2} \phi+\left|\begin{array}{c}
\rho_{1} \rho_{2} \\
\sigma_{1} \sigma_{2}
\end{array}\right| \cos \phi \sin \phi+\left|\begin{array}{c}
\rho_{3} \rho_{2} \\
\sigma_{3} \sigma_{2}
\end{array}\right| \sin ^{2} \phi=0 .
$$

The roots of this equation for $\phi$ give the conjugate directions, and formula (2.26) can be proved easily from (2.29) and (2.22).

A consequence of (2.26) is that the two conjugate directions are orthogonal to each other if and only if $(G)$ degenerates into a line segment; in particular, the conjugate directions are indeterminate if and only if this line segment passes through $P$. This is in accordance with Theorem 2.1.

The Kommerell conic $(K)$ is the locus of the point, apart from $P$, at which the normal plane of $V_{2}$ at $P$ is intersected by the neighboring normal planes. It can be shown that the polar line (with respect to the unit circle in the normal plane) of the point $\phi$ on $(G)$ touches $(K)$ at the point that is the intersection of the normal plane at $P$ by the normal plane at a point near $P$ in the direction $\phi: i^{a}=i_{(1)}^{a} \cos \phi+i_{(2)}^{a} \sin \phi .(K)$ is therefore the polar reciprocal of $(G)$ with respect to the unit circle at $P$, and its equation is

$$
\left(\rho_{1} z+\sigma_{1} t-1\right)\left(\rho_{2} z+\sigma_{2} t-1\right)=\left(\rho_{3} z+\sigma_{3} t\right)^{2} .
$$

We point out, for future reference, that when $(G)$ has a focus at $P,(K)$ is a circle, and conversely.

Although in many literatures the Kommerell conic has been the central figure in the curvature theory of $V_{\mathbf{2}}$ in $R_{\mathbf{4}}$, it will not appear again in this paper, the curvature conic $(G)$ having taken its place.

3. Fundamental equations for $V_{2}$ in $S_{4}$. We now continue the theory of $V_{2}$ in $S_{4}$ which was commenced in $\$ 2.1$. The fundamental equations for a $V_{2}$ in $S_{4}$ consist of the following two groups of equations (Schouten-Struik [15, chaps. 2-3]; Eisenhart [8, chaps. 4-5]):

$$
\begin{aligned}
& \partial_{c} y^{*}=B_{c}^{*}, \\
& \partial_{c} B_{b}^{\alpha}=\Gamma_{c b}^{a} B_{a}^{\alpha}+\Gamma_{\mu \lambda}^{\alpha} B_{c}^{\mu} B_{b}^{\lambda}+h_{c b}^{(3)} i_{(3)}^{*}+h_{c b}^{(4)} i_{(4)}^{k}, \\
& \partial_{c} i_{(3)}^{k}=-\Gamma_{\mu \lambda}^{\alpha} B_{c}^{\mu} i_{(3)}^{\lambda}-v_{c} i_{(4)}^{*}-h_{c}^{(3) b} B_{b}^{\alpha} \text {, } \\
& \partial_{c} i_{(4)}^{k}=-\Gamma_{\mu \lambda}^{k} B_{c}^{\mu} i_{(4)}^{\lambda}+v_{c} i_{(3)}^{k}-h_{c}^{(4) b} B_{b}{ }^{k} \text {; }
\end{aligned}
$$




$$
\begin{gathered}
2(C-K)=\sum_{p=-3}^{4}\left[h_{a}^{(p) b} h_{b}^{(p) a}-\left(h_{a}^{(p) a}\right)^{2}\right], \\
h_{a[b, c]}^{(3)}=-h_{a\left[b v_{c}\right]}^{(4)}, \quad h_{a[b, c]}^{(4)}=h_{a\left[b v_{c]}\right]}^{(3)}, \quad v_{[b, c]}=h_{a[b}^{(4)} h_{c]}^{(3) a}{ }^{(6)} ;
\end{gathered}
$$

where

$$
h_{c b}^{(p)}=-B_{c}^{\lambda} B_{b}^{k} \nabla_{\lambda} i_{k}^{(p)}, \quad v_{c}=B_{c}^{\lambda} i_{(3)}^{k} \nabla_{\lambda} i_{k}^{(4)},
$$

$C=-R / 12$ is the (constant) scalar curvature of $S_{4}$ (in the sense of Schouten $\left({ }^{7}\right)$ ), and the comma denotes covariant differentiation with respect to the fundamental tensor (2.2) of $V_{2}$. We observe that these fundamental equations, taken as a whole, are independent of the choice of the mutually orthogonal unit normal vectors $i_{(3)}^{\kappa}, i_{(4)}^{\kappa}$ to $V_{2}$.

Eisenhart $\left[8\right.$, p. 212] proved that given tensors $g_{c b}, h_{c b}^{(3)}, h_{c b}^{(\mathbf{( s )}}$ and a vector $v_{c}$ satisfying (3.2), a $V_{2}$ in $S_{4}$ is determined (by (3.1)) to within a "motion" in $S_{4}$ such that its first and second fundamental tensors are the given tensors.

By a motion in $S_{4}$ we mean a transformation of the points in $S_{4}$ which preserves the linear element of $S_{4}$. In particular, a motion in a Euclidean space $R_{4}$ reduces to rotations and reflections. Hereafter, we shall call two $V_{2}$ in $S_{4}$ congruent if one is obtainable from the other by a motion in $S_{4}$.

As in $\$ 2.3$, we express $h_{c b}^{(3)}, h_{c b}^{(4)}, v_{c}, i^{a}$ in terms of two mutually orthogonal unit vectors $i_{(1)}^{a}, i_{(2)}^{a}$ in $V_{2}$ :

$$
\begin{aligned}
h_{c b}^{(3)} & =\rho_{1} i_{c}^{(1)} i_{b}^{(1)}+\rho_{2} i_{c}^{(2)} i_{b}^{(2)}+\rho_{3}\left(i_{c}^{(1)} i_{b}^{(2)}+i_{c}^{(2)} i_{b}^{(1)}\right), \\
h_{c b}^{(4)} & =\sigma_{1} i_{c}^{(1)} i_{b}^{(1)}+\sigma_{2} i_{c}^{(2)} i_{b}^{(2)}+\sigma_{3}\left(i_{c}^{(1)} i_{b}^{(2)}+i_{c}^{(2)} i_{b}^{(1)}\right), \\
v_{c} & =\nu_{1} i_{c}^{(1)}+\nu_{2} i_{c}^{(2)}, \quad i^{a}=i_{(1)}^{a} \cos \phi+i_{(2)}^{a} \sin \phi .
\end{aligned}
$$

Then the equations of $(G)$, referred to the rectangular coordinate axes $i_{(3)}^{k}, i_{(4)}^{k}$, are

$$
\begin{aligned}
& z=2^{-1}\left(\rho_{1}+\rho_{2}\right)+2^{-1}\left(\rho_{1}-\rho_{2}\right) \cos 2 \phi+\rho_{3} \sin 2 \phi, \\
& t=2^{-1}\left(\sigma_{1}+\sigma_{2}\right)+2^{-1}\left(\sigma_{1}-\sigma_{2}\right) \cos 2 \phi+\sigma_{3} \sin 2 \phi,
\end{aligned}
$$

and the fundamental equations (3.2) take the invariant form

$$
\begin{gathered}
C-K=\rho_{3}^{2}-\rho_{1} \rho_{2}+\sigma_{3}^{2}-\sigma_{1} \sigma_{2}, \\
\delta_{2} \rho_{1}-\delta_{1} \rho_{3}+\beta\left(\rho_{1}-\rho_{2}\right)-2 \alpha \rho_{3}-\nu_{1} \sigma_{3}+\nu_{2} \sigma_{1}=0, \\
\delta_{1} \rho_{2}-\delta_{2} \rho_{3}-\alpha\left(\rho_{1}-\rho_{2}\right)-2 \beta \rho_{3}-\nu_{2} \sigma_{3}+\nu_{1} \sigma_{2}=0, \\
\delta_{2} \sigma_{1}-\delta_{1} \sigma_{3}+\beta\left(\sigma_{1}-\sigma_{2}\right)-2 \alpha \sigma_{3}+\nu_{1} \rho_{3}-\nu_{2} \rho_{1}=0, \\
\delta_{1} \sigma_{2}-\delta_{2} \sigma_{3}-\alpha\left(\sigma_{1}-\sigma_{2}\right)-2 \beta \sigma_{3}+\nu_{2} \rho_{3}-\nu_{1} \rho_{2}=0, \\
\delta_{2} \nu_{1}-\delta_{1} \nu_{2}+\beta \nu_{1}-\alpha \nu_{2}+\left(\rho_{1}-\rho_{2}\right) \sigma_{3}-\left(\sigma_{1}-\sigma_{2}\right) \rho_{3}=0,
\end{gathered}
$$

( $\left.{ }^{\circ}\right)$ Here we write, for example, $v_{b, e}-v_{c, b}=v_{[b, e]}$.

(7) Eisenhart [8] called $R$ the scalar curvature. 
where

$$
\alpha=-i_{(1)}^{b} i_{(2)}^{c} i_{b, c}^{(2)}, \quad \beta=-i_{(2)}^{b} i_{(1)}^{c} i_{b, c}^{(1)}, \quad \delta_{1} f=i_{(1)}^{c} f_{, c}, \quad \delta_{2} f=i_{(2)}^{c} f_{, c}
$$

are as defined in $\$ 1.2$. Formulas (3.6) are obtained after a straightforward but rather lengthy calculation.

If the conic $(G)$ and its relation to the point $P$ are of certain nature all over $V_{2}$, we can make use of this particular nature to simplify the equations of $(G)$, and consequently the fundamental equations (3.6), by suitable choice of $i_{(3)}^{k}$ and $i_{(1)}^{a}$. This choice is evidently equivalent to the choice of the $z$-axis in the plane of $(G)$ and the position of the point $(\phi=0)$ on $(G)$. Consider, for example, the special $V_{2}$ for which (1) $P$ is the center of $(G),(2)(G)$ is a circle, (3) $P$ is a focus of $(G),(4) P$ lies on $(G)$, and (5) $(G)$ is a line segment subtending a right angle at $P$, respectively. By choosing the $z$-axis and the point $(\phi=0)$ on $(G)$ as in the following figures,
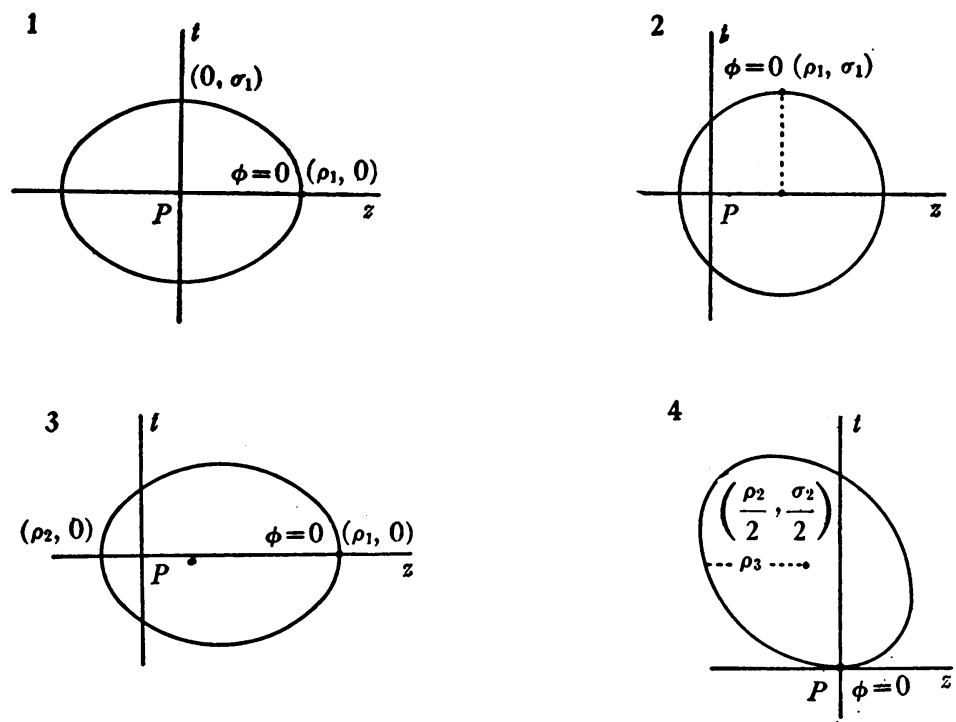

5

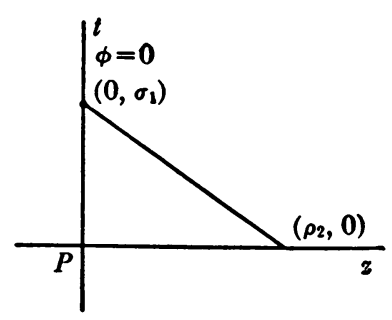

the simplified equations of $(G)$ and the equations satisfied by the $\rho$ 's and $\sigma$ 's for the respective cases are: 
$(3.7)_{1} \quad z=\rho_{1} \cos 2 \phi, \quad t=\sigma_{3} \sin 2 \phi ; \quad \rho_{2}=-\rho_{1}, \quad \rho_{3}=\sigma_{1}=\sigma_{2}=0$.

$(3.7)_{2} z=\rho_{1}+\sigma_{1} \sin 2 \phi, t=\sigma_{1} \cos 2 \phi ; \sigma_{2}=-\sigma_{1}, \rho_{3}=\sigma_{1}, \rho_{2}=\rho_{1}, \sigma_{3}=0$.

$(3.7)_{3}$ $z=2^{-1}\left(\rho_{1}+\rho_{2}\right)+2^{-1}\left(\rho_{1}-\rho_{2}\right) \cos 2 \phi, \quad t=\left(-\rho_{1} \rho_{2}\right)^{1 / 2} \sin 2 \phi ;$ $\rho_{3}=\sigma_{1}=\sigma_{2}=0, \quad \sigma_{3}^{2}=-\rho_{1} \rho_{2}$. $z=2^{-1} \rho_{2}(1-\cos 2 \phi)+\rho_{3} \sin 2 \phi, \quad t=2^{-1} \sigma_{2}(1-\cos 2 \phi) ;$ $\rho_{1}=\sigma_{1}=\sigma_{3}=0$.

$(3.7)_{5} z=2^{-1} \rho_{2}(1-\cos 2 \phi), t=2^{-1} \sigma_{1}(1+\cos 2 \phi) ; \rho_{1}=\rho_{3}=\sigma_{2}=\sigma_{3}=0$.

It is seen that for any special $V_{2}$ there is a system of equations (3.7) in the $\rho$ 's and $\sigma$ 's analogous to the second set of equations in $(3.7)_{1}$ for case 1 . Then from the above-mentioned Eisenhart's theorem it follows that the existence of this particular $V_{2}$ depends on the consistency of equations (3.6) and (3.7) in $i_{(c)}^{a}$ and the $\rho$ 's, $\sigma$ 's, $\nu$ 's. If these equations are consistent, the $V_{2}$ in question exists, and all its intrinsic properties can be derived from (3.6) and (3.7). In particular, when the solution of the latter equations can be found explicitly, the tensors $g_{c b}, h_{c b}^{(3)}, h_{c b}^{(4)}, v_{c}$ are determined. And then equations (3.1) give the manner of imbedding of this $V_{2}$ in $S_{4}$. In the case where (3.1) can actually be solved, we obtain the finite equations of $V_{2}$.

Since for any $V_{2}$ in $S_{4}$, the second fundamental tensors $h_{c b}^{(p)}$ admit an orthogonal transformation, which arises from the permissible change of the mutually orthogonal unit normals $i_{(p)}^{\kappa}$ to $V_{2}$, the arbitrariness of the imbedding of a $V_{2}$ in $S_{4}$, to within motion in $S_{4}$, is equal to the arbitrariness of the solution of (3.6) and (3.7) for the $i_{(c)}^{a}, \rho$ 's, $\sigma$ 's and $\nu$ 's minus the arbitrariness of $h_{c b}^{(p)}$ (that is, of the $\left.i_{(p)}^{k}\right)$ in compliance with (3.7). It is obvious from the very origin of (3.7) that the latter equations usually limit the arbitrariness of $i_{(p)}^{x}$ to mere changes of signs. In fact, the only case to the contrary is when $(G)$ is a circle with center at $P$, that is, when $\sigma_{3}=\rho_{1}$ in $(3.7)_{1}$; this is the case of an $R$-surface (cf. §4).

The rest of this paper will be devoted to the study of some special types of surfaces, particularly the above-mentioned ones.

4.1. Minimal surfaces in $S_{4}$. A minimal $V_{2}$ in $S_{4}$ is a $V_{2}$ whose mean normal curvature vector is everywhere zero, that is, whose curvature conic $(G)$ at each point $P$ always has its center at $P$. A minimal $V_{2}$ is called an $R$-surface (a plane surface $\left(^{8}\right)$ ) if its curvature conic is always a circle of nonzero (zero) radius. A minimal $V_{2}$ that is not an $R$-surface nor a plane surface is called a general minimal surface.

The $R$-surface in an $R_{4}$ was first studied by Kwietniewski [12] and Kommerell [11], and the minimal surface in $R_{4}$ by Eisenhart [6]. The $R$-surface in an $S_{4}$ was later studied by Borůvka [2]. The first three authors based

(8) In general, a subspace in a Riemannian $n$-space $V_{n}$ is said to be plane if its normal curvature vector in $V_{n}$ is zero for every direction at every point. 
their studies on the finite equations of the surface, which are available for a minimal $V_{2}$ in $R_{4}$. The finite equations of a minimal $V_{2}$ in an $S_{4}$, however, are still to be found, and Borůvka used a method which agrees in spirit with the one we outlined in the preceding section. In what follows we shall obtain a necessary and sufficient condition in invariant form for a linear element $d s^{2}$ to be one of a minimal $V_{2}$ (or $R$-surface) in $S_{4}$. We shall give also generalizations to some of Kommerell and Eisenhart's results.

4.2. Fundamental equations. $R$-surface. For a minimal $V_{2}, P$ is the center of $(G)$ and, therefore, with suitable choice of $i_{(3)}^{k}, i_{(1)}^{a}$, the equations of the conic $(G)$ are (cf. $\left.(3.7)_{1}\right)$

$$
z=\rho_{1} \cos 2 \phi, \quad t=\sigma_{3} \sin 2 \phi,
$$

so that

$$
\begin{aligned}
\rho_{2} & =-\rho_{1}, & \rho_{3} & =\sigma_{1}=\sigma_{2}=0 \\
h_{c b}^{(3)} & =\rho_{1}\left(i_{c}^{(1)} i_{b}^{(1)}-i_{c}^{(2)} i_{b}^{(2)}\right), & h_{c b}^{(4)} & =\sigma_{3}\left(i_{c}^{(1)} i_{b}^{(2)}+i_{c}^{(2)} i_{b}^{(1)}\right) .
\end{aligned}
$$

Then the fundamental equations (3.6) become

$$
\begin{gathered}
C-K=\rho_{1}^{2}+\sigma_{3}^{2} \\
\delta_{2} \rho_{1}+2 \beta \rho_{1}-\nu_{1} \sigma_{3}=0, \quad-\delta_{1} \rho_{1}-2 \alpha \rho_{1}-\nu_{2} \sigma_{3}=0 \\
-\delta_{1} \sigma_{3}-2 \alpha \sigma_{3}-\nu_{2} \rho_{1}=0, \quad-\delta_{2} \sigma_{3}-2 \beta \sigma_{3}+\nu_{1} \rho_{1}=0 \\
\delta_{2} \nu_{1}-\delta_{1} \nu_{2}+\beta \nu_{1}-\alpha \nu_{2}+2 \rho_{1} \sigma_{3}=0 .
\end{gathered}
$$

Eliminations of $\nu_{2}$ from $(4.4)_{2,3}$ (that is, the second and third equations in (4.4)) and of $\nu_{1}$ from (4.4) $)_{1,4}$ give, respectively,

$$
\delta_{1}\left(\rho_{1}^{2}-\sigma_{3}^{2}\right)+4 \alpha\left(\rho_{1}^{2}-\sigma_{3}^{2}\right)=0, \quad \delta_{2}\left(\rho_{1}^{2}-\sigma_{3}^{2}\right)+4 \beta\left(\rho_{1}^{2}-\sigma_{3}^{2}\right)=0 .
$$

Consider first the $R$-surface, for which $(G)$ is a circle of nonzero radius. In this case $\sigma_{3}= \pm \rho_{1} \neq 0$. But we may suppose that

$$
\sigma_{3}=\rho_{1} \equiv \rho \neq 0,
$$

after reversing the sense of $i_{(2)}^{a}$ if necessary. Then (4.6) are identically satisfied, and (4.3) and (4.4) become

$$
\begin{gathered}
C-K=2 \rho^{2}, \\
\nu_{1}=\delta_{2} \log \rho+2 \beta, \quad-\nu_{2}=\delta_{1} \log \rho+2 \alpha .
\end{gathered}
$$

If we substitute these values for $\nu_{1}, \nu_{2}$ in (4.5), we have

$$
\left(\delta_{1} \delta_{1}+\delta_{2} \delta_{2}+\alpha \delta_{1}+\beta \delta_{2}\right) \log \rho+2 \rho^{2}+2\left(\delta_{1} \alpha+\delta_{2} \beta+\alpha^{2}+\beta^{2}\right)=0 .
$$

Using (1.5), (1.6) and the value of $\rho$ from (4.7), this becomes

$$
\Delta_{2} \log (C-K)+2 C-6 K=0,
$$


where $\Delta_{2}$ indicates the second differential parameter with respect to the linear element $d s^{2}$ of $V_{2}$. For the $R$-surface in an $R_{4}, C=0$ and (4.9) reduces to

$$
\Delta_{2} \log (-K)-6 K=0 .
$$

Equation (4.9), which is in an invariant form, is a necessary condition for a $d s^{2}$ to be the linear element of an $R$-surface in an $S_{4}$. To prove the sufficiency of this condition, let there be given a $d s^{2}$ satisfying it with some constant $C$. We may choose any mutually orthogonal unit vectors $i_{(1)}^{a}, i_{(2)}^{a}$ in $V_{2}$, and, after having obtained a value for $\rho$ from (4.7), we find from (4.8) the values for $\nu_{1}, \nu_{2}$. Then we have a solution of the fundamental equations (4.3)(4.5), and therefore an $R$-surface in $S_{4}$ with this $d s^{2}$ as linear element. Hence we have the following theorem.

THEOREM 4.1. $A d s^{2}$ is the linear element of an $R$-surface in an $S_{4}$ if and only if (4.9) is satisfied for some constant $C$. Then $C$ is the (constant) scalar curvature of the $S_{4} . A d s^{2}$ is the linear element of an $R$-surface in an $R_{4}$ if and only if (4.10) is satisfied.

If $K=$ const., (4.9) and (4.7) reduce to $K=C / 3=\rho^{2}>0$. Hence, we have proved in a different way the following result due to Borůvka [2]:

THEOREM 4.2. In an $S_{4}$ of scalar curvature $C>0$, there exists only one type of $R$-surface of constant Gaussian curvature $K$. For this, $K=C / 3$, and the curvature circle $(G)$ is of constant radius $(K)^{1 / 2}$ all over the surface. In an $R_{4}$ an $R$-surface has negative Gaussian curvature, which cannot be constant.

The last part of the theorem follows at once from (4.7) and (4.10).

Kommerell $[11, \S 11]$ proved the rigidity of an $R$-surface in an $R_{4}$. We shall now prove this result for the more general case of an $R$-surface in an $S_{4}$, using a different method.

Theorem 4.3. If two $R$-surfaces $V_{2}, \bar{V}_{2}$ in an $S_{4}$ are applicable, then they are congruent.

Proof. We use a dash to indicate quantities and equations belonging to $\bar{V}_{2}$ and suppose that the unit vectors $i_{(p)}^{\kappa}, i_{(c)}^{a}, i_{(p)}^{\kappa}, i_{(c)}^{a}$ have been so chosen that equations (4.1), $(4.1)_{a},\left(4.1^{-}\right),\left(4.1^{-}\right)_{a}$ are satisfied. Then since $d \bar{s}^{2}=d s^{2}$, we have from earlier results in this section:

(4.11) $C-K=2 \rho^{2}$,

$$
h_{c b}^{(3)}=\rho\left(i_{c}^{(1)} i_{b}^{(1)}-i_{c}^{(2)} i_{b}^{(2)}\right),
$$

$$
\begin{aligned}
h_{c b}^{(4)} & =\rho\left(i_{c}^{(1)} i_{b}^{(2)}+i_{c}^{(2)} i_{b}^{(1)}\right), \\
v_{c} & =\left(\delta_{2} \log \rho+2 \beta\right) i_{c}^{(1)} \\
& -\left(\delta_{1} \log \rho+2 \alpha\right) i_{c}{ }^{(2)},
\end{aligned}
$$

$$
\begin{aligned}
C-K & =2 \bar{\rho}^{2}, \\
h_{c b}^{(3)} & =\bar{\rho}\left(\bar{i}_{c}^{(1)} \bar{i}_{b}^{(1)}-\bar{i}_{c}^{(2)} \bar{i}_{b}^{(2)}\right), \\
\bar{h}_{c b}^{(4)} & =\bar{\rho}\left(\bar{i}_{c}^{(1)} \bar{i}_{b}^{(2)}+\bar{i}_{c}^{(2)} \bar{i}_{b}^{(1)}\right), \\
\bar{v}_{c} & =\left(\bar{\delta}_{2} \log \bar{\rho}+2 \bar{\beta}\right) \bar{i}_{c}^{(1)} \\
& -\left(\bar{\delta}_{1} \log \bar{\rho}+2 \bar{\alpha}\right) \bar{i}_{c}^{(2)} .
\end{aligned}
$$


Let us express $\bar{i}_{c}^{(1)}, \bar{i}_{c}^{(2)}$ in terms of $i_{c}^{(1)}, i_{c}^{(2)} ;$ thus

$$
i_{c}^{(1)}=i_{c}^{(1)} \cos \theta+i_{c}^{(2)} \sin \theta, \epsilon i_{c}^{(2)}=-i_{c}^{(1)} \sin \theta+i_{c}^{(2)} \cos \theta(\epsilon= \pm 1) .
$$

From (4.11) and $\left(4.11^{-}\right)$we have

$$
\bar{\rho}=\epsilon^{\prime} \rho \quad\left(\epsilon^{\prime}= \pm 1\right) .
$$

If we use (4.14) and (4.15) in (4.12-) and (4.13-), and then take account of (4.12) and (4.13), we have by straightforward calculation:

$$
\begin{aligned}
\bar{h}_{c b}^{(3)} & =\epsilon^{\prime}\left(h_{c b}^{(3)} \cos 2 \theta+h_{c b}^{(4)} \sin 2 \theta\right), \\
\bar{h}_{c b}^{(4)} & =\epsilon \epsilon^{\prime}\left(-h_{c b}^{(3)} \sin 2 \theta+h_{c b}^{(4)} \cos 2 \theta\right), \\
\bar{v}_{c} & =\epsilon\left[v_{c}-2\left(i_{c}^{(1)} \delta_{1} \theta+i_{c}^{(2)} \delta_{2} \theta\right)\right]=\epsilon\left[v_{c}-B_{c}^{\lambda} \nabla_{\lambda}(2 \theta)\right] .
\end{aligned}
$$

Let us now replace the normal vectors $i_{(3)}^{\kappa}, i_{(4)}^{\kappa}$ to $V_{2}$ by the following mutually orthogonal unit vectors

$$
' i_{(3)}{ }^{k}=\epsilon^{\prime}\left(i_{(3)}{ }^{k} \cos 2 \theta+i_{(4)}^{k} \sin 2 \theta\right), \quad{ }^{\prime}{ }_{(4)}{ }^{k}=\epsilon \epsilon^{\prime}\left(-i_{(3)}{ }^{k} \sin 2 \theta+i_{(4)}{ }^{k} \cos 2 \theta\right),
$$

which are normal to $V_{2}$. Then the corresponding tensors ${ }^{\prime} h_{c b}^{(3)},{ }^{\prime} h_{c b}^{(4)},{ }^{\prime} v_{c}$ for $V_{2}$ are

$$
\begin{aligned}
{ }^{\prime} h_{c b}^{(3)} & =-B_{c}^{\lambda} B_{b}^{\alpha} \nabla_{\lambda}^{\prime} i_{k}^{(3)}=\epsilon^{\prime}\left(h_{c b}^{(3)} \cos 2 \theta+h_{c b}^{(4)} \sin 2 \theta\right), \\
{ }^{\prime} h_{c b}^{(4)} & =-B_{c}^{\lambda} B_{b}^{\alpha} \nabla_{\lambda}^{\prime} i_{k}^{(4)}=\epsilon \epsilon^{\prime}\left(-h_{c b}^{(3)} \sin 2 \theta+h_{c b}^{(4)} \cos 2 \theta\right), \\
{ }^{\prime} v_{c} & =\epsilon\left[v_{c}-B_{c}^{\lambda} \nabla_{\lambda}(2 \theta)\right] .
\end{aligned}
$$

Since these tensors are identical with $\bar{h}_{c b}^{(3)}, \bar{h}_{c b}^{(4)}, \bar{v}_{c}$, respectively, we may conclude by Eisenhart's theorem that $\bar{V}_{2}$ differs from $V_{2}$ only by a motion in $S_{4}$.

4.3. General minimal surface. For a general minimal $V_{2}$ (cf. §4.1), we have (4.1)-(4.5) and $\rho_{1}^{2} \neq \sigma_{3}^{2}$. In conformity with (4.3) we write

$$
\rho_{1}=(C-K)^{1 / 2} \cos w, \quad \sigma_{3}=(C-K)^{1 / 2} \sin w,
$$

where $w$ is a scalar, so that

$$
\rho_{1}^{2}-\sigma_{3}^{2}=(C-K) \cos 2 w \neq 0 .
$$

We observe that since $\rho_{1}, \sigma_{3}$ are numerically equal to the semi-axes of the conic $(G)$, and $\sigma_{3} / \rho_{1}=\tan w$, $w$ determines the shape of $(G)$.

On account of (4.18), equations (4.6) become.

(4.20) $\delta_{1} \log [(C-K) \cos 2 w]+4 \alpha=0, \delta_{2} \log [(C-K) \cos 2 w]+4 \beta=0$.

Or, if we write for convenience

$$
\begin{aligned}
& \xi=4^{-1} \log [(C-K) \cos 2 w], \\
& \delta_{1} \xi+\alpha=0, \quad \delta_{2} \xi+\beta=0 .
\end{aligned}
$$


The integrability condition of $\left(4.20^{\prime}\right)$ is

$$
\delta_{2} \alpha-\delta_{1} \beta=0 \text {. }
$$

This shows that the congruences $i_{(1)}^{a}, i_{(2)}^{a}$ form an isometric net in $V_{2}$. The curves of the congruences $i_{(1)}^{a}, i_{(2)}^{a}$ are called the lines of curvature of the general minimal $V_{2}$ in $S_{4}$ for the reason that the normal curvature of $V_{2}$ in $S_{4}$ has stationary values in the directions $i_{(1)}^{a}, i_{(2)}^{a}$, being equal to the semi-axes of $(G)$. Hence we have the following theorem.

THEOREM 4.4. The lines of curvature of a general minimal $V_{2}$ in $S_{4}$ form an isometric net.

To eliminate $i_{(1)}^{a}, i_{(2)}^{a}$ from $\left(4.20^{\prime}\right)$, we need only calculate $\Delta_{2} \xi$, thus

$$
\Delta_{2} \xi=\left(\delta_{1} \delta_{1}+\delta_{2} \delta_{2}+\alpha \delta_{1}+\beta \delta_{2}\right) \xi=-\left(\delta_{1} \alpha+\delta_{2} \beta+\alpha^{2}+\beta^{2}\right)=K .
$$

Therefore,

$$
\Delta_{2} \xi=K
$$

or, substituting back the value (4.21) for $\xi$,

$$
\Delta_{2} \log [(C-K) \cos 2 w]=4 K .
$$

Let us now solve $(4.4)_{1,2}$ for $\nu_{1}, \nu_{2}$, then use the values of $\rho_{1}$ and $\sigma_{3}$ from (4.18), and finally eliminate $(C-K)$ by means of (4.20). Then the result is, after simplification,

$$
\begin{aligned}
& \nu_{1}=\frac{\delta_{2} w}{\cos 2 w}=2^{-1} \delta_{2} \log \frac{1+\sin 2 w}{\cos 2 w}, \\
& \nu_{2}=\frac{-\delta_{1} w}{\cos 2 w}=-2^{-1} \delta_{1} \log \frac{1+\sin 2 w}{\cos 2 w} .
\end{aligned}
$$

Using these in (4.5), we get

$$
\Delta_{2} \log \frac{1+\sin 2 w}{\cos 2 w}+2(C-K) \sin 2 w=0 .
$$

Equations (4.23) and (4.25) can be rewritten as

$$
\begin{aligned}
\Delta_{2} \log \cos 2 w & =\Delta_{2} \log (1+\sin 2 w)+2(C-K) \sin 2 w \\
& =-\left[\Delta_{2} \log (C-K)-4 K\right] .
\end{aligned}
$$

We now prove the following theorem.

TheOREM 4.5. A necessary and sufficient condition for $a d s^{2}$ to be the linear element of a general minimal $V_{2}$ in an $S_{4}$ of scalar curvature $C$ is that there exist a solution $w$ of the equations (4.23) and (4.25). If the condition is satisfied, then w determines the shape of the curvature conic of $V_{2}$ in $S_{4}$. 
Before we proceed to prove this theorem, we remark that it is highly desirable to be able to eliminate $w$ from (4.26), thus replacing the condition (4.26) by one that involves only invariant quantities for $d s^{2}$. But so far the author has not succeeded in doing this.

Proof. We have seen that the condition stated in the theorem is necessary. To prove that it is also sufficient, we need only show that given a $d s^{2}$ satisf ying the condition and given a solution $w$ of (4.23) and (4.25), there exist two mutually orthogonal unit vectors $i_{(1)}^{a}, i_{(2)}^{a}$ satisfying (4.20). For, if this is true, we may obtain $\rho_{1}, \sigma_{3}$ from (4.18), and $\nu_{1}, \nu_{2}$ from (4.24). Then, on account of (4.25), these scalars, together with $i_{(1)}^{a}, i_{(2)}^{a}$, satisfy the equations (4.3)-(4.5).

Let $i_{(1)}^{* a}, i_{(2)}^{* a}$ be an isometric net of $d s^{2}$, and indicate quantities belonging to them by a star. Then

$$
\delta_{2}^{*} \alpha^{*}-\delta_{1}^{*} \beta^{*}=0,
$$

and consequently, there exists a scalar $\xi^{*}$ satisfying

$$
\delta_{1}^{*} \xi^{*}+\alpha^{*}=0, \quad \delta_{2}^{*} \xi^{*}+\beta^{*}=0 .
$$

This $\xi^{*}$ is a solution of $\left(4.23^{\prime}\right)$ for $\xi$, as is evident from the way this equation was derived. Now by hypothesis (4.23), the $\xi$ defined by (4.21) is also a solution of $\left(4.23^{\prime}\right)$. Therefore

$$
\xi=\xi^{*}+\psi,
$$

where $\psi$ is a scalar such that

$$
\Delta_{2} \psi=\left(\delta_{1}^{*} \delta_{1}^{*}+\delta_{2}^{*} \delta_{2}^{*}+\alpha^{*} \delta_{1}^{*}+\beta^{*} \delta_{2}^{*}\right) \psi=0 .
$$

We wish to find the most general pair of mutually orthogonal unit vectors

$$
\begin{aligned}
& i_{(1)}^{a}=i_{(1)}^{* a} \cos \theta+i_{(2)}^{* a} \sin \theta, \\
& i_{(2)}^{a}=\epsilon\left(-i_{(1)}^{* a} \sin \theta+i_{(2)}^{* a} \cos \theta\right) \quad(\epsilon= \pm 1)
\end{aligned}
$$

satisfying $\left(4.20^{\prime}\right)$. Written in terms of $i_{(1)}^{* a}, i_{(2)}^{* a}$, equations $\left(4.20^{\prime}\right)$ are (cf. (1.8)(1.10))

$$
\begin{aligned}
\epsilon\left(-\sin \theta \delta_{1}^{*}+\cos \theta \delta_{2}^{*}\right) \xi-\epsilon\left[\left(-\beta^{*}+\delta_{1}^{*} \theta\right) \cos \theta+\left(\alpha^{*}+\delta_{2}^{*} \theta\right) \sin \theta\right]=0, \\
\left(\cos \theta \delta_{1}^{*}+\sin \theta \delta_{2}^{*}\right) \xi+\left[-\left(-\beta+\delta_{1}^{*} \theta\right) \sin \theta+\left(\alpha^{*}+\delta_{2}^{*} \theta\right) \cos \theta\right]=0,
\end{aligned}
$$

which are equivalent to

$$
\delta_{1}^{*} \xi+\alpha^{*}+\delta_{2}^{*} \theta=0, \quad \delta_{2}^{*} \xi+\beta^{*}-\delta_{1}^{*} \theta=0 .
$$

These become, on account of (4.27) and (4.20*),

$$
\delta_{1}^{*} \psi+\delta_{2}^{*} \theta=0, \quad \delta_{2}^{*} \psi-\delta_{1}^{*} \theta=0 .
$$

The integrability condition of (4.30) for $\theta$ is (4.28), and is therefore satisfied. Hence (4.30) admits a unique solution, to within an additive constant, for $\theta$. 
In other words, for the solution (4.21) of $\left(4.23^{\prime}\right)$, equations $\left(4.20^{\prime}\right)$ have solutions for $i_{(1)}^{a}, i_{(2)}^{a}$, and, if $i_{(1)}^{* a}, i_{(2)}^{* a}$ is a solution, the most general solution is (4.29) with $\theta=$ const. Theorem 4.5 is thus completely proved.

Incidentally we observe that all the results so far obtained for the general minimal $V_{z}$ remain true when $\sigma_{3}=0$, that is, when $w=0$. But $w=0$ implies that $h_{c b}^{(4)}=0$ and $v_{c}=0$, as follows from (4.1) and (4.24). Therefore, this is the case of a minimal surface in an $S_{3}$ of scalar curvature $C$ (cf. (7.2) and Schouten-Struik [15, p. 150]). Since $\rho_{1} \neq 0$ by (4.19), this minimal surface in $S_{3}$ is not a plane surface. Hence we have from (4.26) the following corollary to Theorem 4.5:

CoRollary 4.5. A necessary and sufficient condition for a $d s^{2}$ to be the linear element of a non-plane minimal surface in an $S_{3}$ of scalar curvature $C$ is that $\Delta_{2} \log (C-K)=4 K$ be satisfied.

This result can of course be proved much more easily by using the fundamental equations (7.2) for a $V_{2}$ in $S_{3}$ and should be compared with one of Ricci's [14, p. 340] which states that a necessary and sufficient condition for a $d s^{2}$ to be the linear element of a $V_{2}$ with constant mean curvature $c$ in an $R_{3}$ is that $\Delta_{2} \log \left(c^{2}-K\right)=4 K$ be satisfied.

By using the last part of the proof of Theorem 4.5 we can prove the following theorem.

THEOREM 4.6. In an $S_{4}$ a general minimal $V_{2}^{*}$ which is not a minimal surface in an $S_{3}$ can be deformed continuously into $\infty^{1}$ non-congruent minimal $V_{2}$ 's with equal curvature conics at corresponding points. Any minimal surface $\bar{V}_{2}$ in $S_{4}$ applicable but not congruent to $V_{2}^{*}$ and with curvature conics equal to those of $V_{2}^{*}$ at corresponding points is congruent to one of these $\infty^{1}$ minimal $V_{2}$ 's. The image on $V_{2}^{*}$ of the lines of curvature of any one of these $V_{2}$ 's makes a constant angle with the lines of curvature of $V_{2}^{*}$.

Proof. In an $S_{4}$ let $V_{2}^{*}$ be a general minimal surface which is not a minimal surface in an $S_{3}$, and let $\bar{V}_{2}$ be another which is applicable but not congruent to $V_{2}{ }^{*}$ such that the curvature conics of $V_{2}{ }^{*}$ and $\bar{V}_{2}$ are equal at corresponding points. We use a star and a dash to indicate the quantities and equations belonging to $V_{2}^{*}, \bar{V}_{2}$, respectively, and suppose that equations $\left(4.1^{*}\right),\left(4.1^{-}\right)$are satisfied. Then since $\rho_{1}^{*}, \sigma_{3}{ }^{*}\left(\bar{\rho}_{1}, \bar{\sigma}_{3}\right)$ are numerically equal to the semi-axes of $\left(G^{*}\right)((\bar{G}))$, the vectors $\bar{i}_{(p)}^{\kappa}$, which have been essentially determined by $\left(4.1^{-}\right)$, can be readjusted so that we have

$$
\bar{w}=w^{*}, \quad \bar{\rho}_{1}=\rho_{1}^{*} \neq 0, \quad \bar{\sigma}_{3}=\sigma_{3}^{*} \neq 0 .
$$

From this and (4.21) we have

$$
\bar{\xi}=\xi^{*} \text {. }
$$

Since $i_{(1)}^{* a}, i_{(2)}^{* a}$ satisfy $\left(4.20^{\prime}\right)$ with $\xi=\xi^{*}$, the most general solution for 
$i_{(1)}^{a}, i_{(2)}^{a}$ of $\left(4.20^{\prime}\right)$ with $\xi=\xi^{*}$ is, as we have seen,

$$
\ddot{i}_{(1)}^{a}=i_{(1)}^{* a} \cos \gamma+i_{(2)}^{* a} \sin \gamma, \quad \epsilon i_{(2)}^{a}=-i_{(1)}^{* a} \sin \gamma+i_{(2)}^{* a} \cos \gamma,
$$

where $\gamma=$ const. and $\epsilon= \pm 1$. The $d s^{2}=d s^{* 2}, w=w^{*}$, and the above $i_{(1)}^{a}, i_{(2)}^{a}$ give a solution of the fundamental equations for the imbedding of a $V_{2}$ with linear element $d s^{2}$ in $S_{4}$ as a minimal $V_{2}$ whose curvature conics are, by (4.18), equal to those of $V_{2}^{*}$ at corresponding points. The second fundamental tensors for $V_{2}$ are, by (4.31) and (4.33),

$$
\begin{aligned}
& h_{c b}^{(3)}=\rho_{1}\left(i_{c}^{(1)} i_{b}^{(1)}-i_{c}^{(2)} i_{b}^{(2)}\right)=h_{c b}^{*(3)} \cos 2 \gamma+h_{c b}^{*(4)} \frac{\rho_{1}^{*}}{\sigma_{3}{ }^{*}} \sin 2 \gamma, \\
& h_{c b}^{(4)}=\sigma_{3}\left(i_{c}^{(1)} i_{b}^{(2)}+i_{c}^{(2)} i_{b}^{(1)}\right)=-h_{c b}^{*(3)} \frac{\sigma_{3}^{*}}{\rho_{1}^{*}} \sin 2 \gamma+h_{c b}^{*(4)} \cos 2 \gamma .
\end{aligned}
$$

To find $v_{c}$, we have from (4.24) and (4.33)

$\nu_{1}=\frac{\delta_{2} w}{\cos 2 w}=\epsilon\left(\nu_{2}^{*} \sin \gamma+\nu_{1}^{*} \cos \gamma\right), \quad \nu_{2}=\frac{-\delta_{1} w}{\cos 2 w}=+\nu_{2}^{*} \cos \gamma-\nu_{1}^{*} \sin \gamma$, and therefore

$$
\nu_{c}=\nu_{1} i_{c}^{(1)}+\nu_{2} i_{c}^{(2)}=\epsilon\left(\nu_{1}^{*} i_{c}^{*(1)}+\nu_{2}^{*} i_{c}^{*(2)}\right)=\epsilon v_{c}^{*} .
$$

Since $\left(\sigma_{3}^{*} / \rho_{1}^{*}\right)^{2} \neq 1$, it is seen from (4.34) that unless sin $2 \gamma=0, h_{c b}^{(3)}$ and $h_{c b}^{(4)}$ cannot be obtained from $h_{c b}^{*(3)}$ and $h_{c b}^{*(4)}$ by a change of $i_{(3)}^{* k}$ and $i_{(4)}^{* k}$. Therefore for any value of $\gamma$ such that $\sin 2 \gamma \neq 0$, the corresponding $V_{2}$ is not congruent to $V_{2}^{*}$.

As a consequence of (4.32), the minimal $V_{2}$ will become the given $\bar{V}_{2}$ for some (constant) value $\bar{\gamma}$ of $\gamma$. And the last part of Theorem 4.6 follows at once from (4.33) with $\gamma=\bar{\gamma}$.

Although direct verification may not be easy, it is conceivable that for the case of an $R_{4}$, the $\infty^{1}$ minimal $V_{2}$ 's in Theorem 4.6 are the associate minimal surfaces to $V_{2}^{*}$ defined by Eisenhart $[6, \S 8]$.

It follows from the preceding results that the problem of the deformation of a general minimal $V_{2}$ in $S_{4}$ now reduces to the following problem: Given a $d s^{2}$ for which equations (4.26) have a known particular solution for $w$, to find the most general solution. The author has not yet been able to solve the latter problem.

4.4. Further consequences of (4.23) and (4.25). Equation (4.25) shows that $w$ cannot be constant. Hence we have the following theorem.

THEOREM 4.7. The curvature conics of an $R$-surface in $S_{4}$ are all circles, and those of a minimal $V_{2}$ in an $S_{3}$, considered as a $V_{2}$ in $S_{4}$, are all line segments. No other minimal $V_{2}$ in an $S_{4}$ has all its curvature conics at different points similar to one another. 
We now prove the following theorem.

THEOREM 4.8. In an $S_{4}$ a plane surface and an $R$-surface with constant Gaussian curvature are the only minimal $V_{2}$ for which the sum of the axes of the curvature conic is constant all over the surface.

Proof. That a plane surface in $S_{4}$ has the property mentioned in the theorem is obvious, and that an $R$-surface with constant Gaussian curvature has the same property follows at once from Theorem 4.3. Excluding these two cases we now suppose that the $\rho_{1}, \sigma_{3}$ for a general minimal $V_{2}$ are both positive (with suitable choice of the senses of $i_{(p)}^{\kappa}$ ). Then we have from (4.18) that the sum of the semi-axes of $G$ ) is

$$
\rho_{1}+\sigma_{3}=(C-K)^{1 / 2}(\cos w+\sin w) .
$$

If this is constant, we have, by squaring, that

$$
(C-K)(1+\sin 2 w)=\text { const. }=A .
$$

Now equation (4.25) can be written as

$$
\Delta_{2} \log \frac{(C-K)(1+\sin 2 w)}{(C-K) \cos 2 w}+2(C-K)(1+\sin 2 w)-2(C-K)=0,
$$

which, in consequence of the preceding equation and (4.23), reduces to

$$
-4 K+2 A-2(C-K)=0, \text { that is, } K=A-C=\text { const. }
$$

This together with (4.36) would require $w$ to be a constant, and that contradicts (4.25). Therefore Theorem 4.8 is proved.

Finally we shall prove the following theorem.

THEOREM 4.9. There exists no minimal $V_{2}$ in $S_{4}$, other than an $R$-surface with constant Gaussian curvature or a minimal $V_{2}$ in an $S_{3}$ considered as a $V_{2}$ in $S_{4}$, for which the curvature conics all have equal area.

Proof. For the first exceptional case stated in the theorem, the area of all the curvature conics is equal to $2 c \pi$ (cf. Theorem 4.3 ); for the second exceptional case, the curvature conic is always a line segment, and therefore its area is zero. Excluding these two cases, if the curvature conic $(G)$ of a general minimal $V_{2}$ in $S_{4}$ has constant area, we have, from (4.18),

$$
\rho_{1} \sigma_{3}=(C-K) \sin w \cos w=\text { const. } \equiv 2^{-1} A,
$$

that is,

$$
(C-K) \sin 2 w=A .
$$

We first note from this that $K$ cannot be constant, otherwise $w=$ const. Equations (4.23), (4.25) can be written, respectively, as 


$$
\begin{gathered}
\Delta_{2} \log [(C-K) \cos 2 w]^{2}=8 K \\
\Delta_{2} \log \left(\frac{1+\sin 2 w}{\cos 2 w}\right)^{2}+4(C-K) \sin 2 w=0 .
\end{gathered}
$$

In virtue of (4.37), these become

$$
\begin{aligned}
\Delta_{2} \log \left[A^{2}-(C-K)^{2}\right] & =8 K, \\
\Delta_{2} \log \frac{A+(C-K)}{A-(C-K)} & =-4 A,
\end{aligned}
$$

which are equivalent to

$$
\Delta_{2} \log (A+C-K)=2(2 K-A), \quad \Delta_{2} \log (A-C+K)=2(2 K+A) .
$$

Since $\Delta_{2} f(K)=f^{\prime} \Delta_{2} K+f^{\prime \prime} \Delta_{1} K$, the preceding equations can be written

$$
\begin{aligned}
& (K-C-A) \Delta_{2} K-\Delta_{1} K=2(2 K-A)(K-C-A)^{2}, \\
& (K-C+A) \Delta_{2} K-\Delta_{1} K=2(2 K+A)(K-C+A)^{2} .
\end{aligned}
$$

Solving these for $\Delta_{2} K, \Delta_{1} K$, we have after simplification

$$
\begin{aligned}
& \Delta_{2} K=\phi(K) \equiv 2\left(5 K^{2}-6 C K+C^{2}+A^{2}\right)=10 K^{2}+\cdots, \\
& \Delta_{1} K=f(K) \equiv 2(3 K-C)\left[(K-C)^{2}-A^{2}\right]=6 K^{3}+\cdots,
\end{aligned}
$$

where the dots indicate terms of lower degrees in $K$.

It is known (cf. Eisenhart [7, p. 97]) that a $d s^{2}$ for which some function $K$ satisfies equations of the form (4.40) can be reduced to

$$
d s^{2}=E d K^{2}+G d t^{2} \equiv \frac{1}{f}\left(d K^{2}+\exp \left(2 \int \phi f^{-1} d K\right) d t^{2}\right) .
$$

For this, the Gaussian curvature $K$ is given by

$$
K=-\frac{1}{(E G)^{1 / 2}}\left[\frac{1}{E^{1 / 2}}\left(G^{1 / 2}\right)^{\prime}\right]^{\prime},
$$

where the prime denotes differentiation with respect to $K$. Substituting here the values of $E, G$, we have after straightforward calculation

$$
K f+\left(\phi-f^{\prime}\right)\left(\phi-2^{-1} f^{\prime}\right)+\left(\phi^{\prime}-2^{-1} f^{\prime \prime}\right) f=0 .
$$

In consequence of (4.40), this is a polynomial equation in $K$, whose term of the highest degree is $10 K^{4}$. Therefore $K$ must be constant. But this has been seen to be impossible. Hence Theorem 4.9 is proved.

4.5. Linear element of a minimal $V_{2}$ in an $R_{4}$. Let us now find the linear element

$$
d s^{2}=2 F d u d v
$$


of a minimal $V_{2}$ in an $R_{4}$. We have for (4.42)

$$
\Delta_{2}=\frac{2}{F} \partial_{v} \partial_{u}, \quad K=-\frac{1}{F} \partial_{v} \partial_{u} \log F
$$

Consider first the case of an $R$-surface. On account of (4.43), equation (4.10) can be written

$$
2 F^{-1} \partial_{v} \partial_{u} \log (-K)=-6 F^{-1} \partial_{v} \partial_{u} \log F,
$$

that is,

$$
\partial_{v} \partial_{u} \log \left(-K F^{3}\right)=0
$$

Therefore

$$
-K F^{3}=U_{1} V_{1},
$$

where $\left({ }^{\circ}\right) U_{1}, V_{1}$ are nonzero functions of $u, v$ alone. Substitution for $K$ from (4.43) gives

$$
\partial_{v} \partial_{u} \log F=U_{1} V_{1} F^{-2} .
$$

Reducing this to Liouville's form by putting $\lambda=F\left(U_{1} V_{1}\right)^{-1 / 2}$, we see that the solution for $F$ is (cf. (1.16), (1.17))

$$
F^{-2}=\frac{U_{2}^{\prime} V_{2}^{\prime}}{U_{1} V_{1}\left(1+U_{2} V_{2}\right)}
$$

where $U_{2}, V_{2}$ are non-constant functions of $u, v$ alone. Therefore

$$
d s^{2}=2\left(1+U_{2} V_{2}\right)\left(\frac{U_{1} V_{1}}{U_{2}^{\prime} V_{2}^{\prime}}\right)^{1 / 2} d u d v,
$$

or, after an obvious transformation on $u, v$ :

$$
d s^{2}=2(1+U V) d u d v,
$$

$U, V$ being arbitrary non-constant functions of $u, v$ alone. Hence we have the following theorem.

THEOREM 4.10. A necessary and sufficient condition for $a d s^{2}$ to be the linear element of an $R$-surface in $R_{4}$ is that it be reducible to the form (4.44).

It can readily be shown that by a suitable change of parameters (4.44) can be reduced to the form

$$
d s^{2}=\left(1+z_{x}^{2}+t_{y}^{2}\right)\left(d x^{2}+d y^{2}\right) \quad\left(z_{x}=\frac{\partial z}{\partial x}, t_{y}=\frac{\partial t}{\partial y}\right),
$$

where $z, t$ are the real and imaginary parts of an arbitrary analytic function

(') The $V_{2}$ here which denotes a function of $v$ should not be confused with the usual $V_{2}$ which denotes a surface. 
$F\left(x+(-1)^{1 / 2} y\right)$. In an $R_{4}$ with rectangular Cartesian coordinates $(X, Y, Z, T)$, (4.45) is the linear element of the surface

$$
X=x, \quad Y=y, \quad Z=z(x, y), \quad T=t(x, y) .
$$

It was in this way that the $R$-surface in $R_{4}$ was first studied by Kwietniewski [12] and Kommerell [11].

It is not difficult to prove from (4.44) or directly from (4.10) the following theorem.

THEOREM 4.11. If $d s^{2}$ is the linear element of an $R$-surface in $R_{4}$, then $e^{2 \sigma} d s^{2}$ will also be one if $\Delta_{2} \sigma=0$.

We now consider the $d s^{2}$ for a general minimal $V_{\mathbf{2}}$ in $R_{\mathbf{4}}$. We rewrite equations (4.23) and (4.25) with $C=0$ as

$$
\Delta_{2} \log \cos 2 w+\Delta_{2} \log (-K)-4 K=0,
$$

$$
\Delta_{2} \log (1+\sin 2 w)+\Delta_{2} \log (-K)-2 K-2 K(1+\sin 2 w)=0 .
$$

On account of (4.43), equation (4.46) can be reduced to

Therefore

$$
\partial_{v} \partial_{u} \log \left(-K F^{2} \cos 2 w\right)=0 .
$$

$$
-K F^{2} \cos 2 w=U(u) V(v) .
$$

Similarly, equation (4.47) can be written

$$
2 F^{-1} \partial_{v} \partial_{u} \log [-F K(1+\sin 2 w)]+2 F^{-1}[-F K(1+\sin 2 w)]=0 .
$$

Omitting the factor $F^{-1}$, this equation takes Liouville's form and therefore has the solution

$$
-F K(1+\sin 2 w)=\frac{2 \bar{U}^{\prime} \bar{V}^{\prime}}{(1+\overline{U V})^{2}} \neq 0,
$$

where $\bar{U}, \bar{V}$ are arbitrary non-constant functions of $u, v$ alone. Eliminating $w$ from (4.48) and (4.49) we get

$$
-F K=\frac{(U V)^{2}}{4 \bar{U}^{\prime} \bar{V}^{\prime}}(1+\overline{U V})^{2} F^{-2}+\frac{\bar{U}^{\prime} \bar{V}^{\prime}}{(1+\overline{U V})^{2}},
$$

which can be written, by using (4.43),

$$
\partial_{v} \partial_{u} \log \left[(1+\overline{U V}) F^{-1}\right]^{2}=-\frac{(U V)^{2}}{4 \bar{U}^{\prime} \bar{V}^{\prime}}\left[(1+\overline{U V}) F^{-1}\right]^{2} .
$$

This again has the form of a Liouville's equation, and therefore its solution is

$$
\frac{(U V)^{2}}{4 \bar{U}^{\prime} \bar{V}^{\prime}}\left[(1+\overline{U V}) F^{-1}\right]^{2}=\frac{2 U^{* \prime} V^{* \prime}}{2\left(1+U^{*} V^{*}\right)^{2}} \neq 0 .
$$


Solving this for $F$, we see that a suitable transformation on $u, v$ will reduce $d s^{2}$ to

$$
d s^{2}=2\left(1+U_{1} V_{1}\right)\left(1+U_{2} V_{2}\right) d u d v,
$$

where $U_{1}, U_{2} ; V_{1}, V_{2}$ are some functions of $u, v$ respectively, none of them being a constant.

When $U_{2}=U_{1}, V_{2}=V_{1}$, formula (4.50) becomes

$$
d s^{2}=2\left(1+U_{1} V_{1}\right)^{2} d u d v,
$$

which happens to be the well known linear element of a non-plane minimal $V_{2}$ in an $R_{3}$ (cf. Eisenhart [7, formula (100), p. 257]). We observe further that when $w=0$, elimination of $K$ from (4.48) and (4.49) also leads to (4.51). Hence we have proved the following theorem.

THEOREM 4.12. A necessary and sufficient condition for a $d s^{2}$ to be the linear element of a general minimal surface in an $R_{4}$ (or a non-plane minimal surface in an $R_{3}$ ) is that it be reducible to the form (4.50)(or (4.51)).

4.6. More properties of the $R$-surface. From the preceding results we have noticed the special position which the $R$-surface occupies among the minimal $V_{2}$ in $S_{4}$. We shall see further that the $R$-surface also occupies a very special position among some other types of surfaces in $S_{4}$. We first prove the following theorem.

THEOREM 4.13. Among the surfaces in $S_{4}$ whose curvature conic at each point $P$ is a circle $(G)$, the one for which the center of $(G)$ is at a constant distance $d$ from $P$ is either an $R$-surface (for which $d=0$ ) or a sphere $\left({ }^{10}\right)$ in a plane $S_{3}$ in $S_{4}$ (for which the circle degenerates into a point). have

Proof. Let $(G)$ be a circle, then by choosing $i_{(3)}^{\kappa}, i_{(1)}^{a}$ suitably (cf. $\left.(4.7)_{2}\right)$ we

$$
\sigma_{2}=-\sigma_{1}, \quad \rho_{3}=\sigma_{1}, \quad \rho_{2}=\rho_{1}, \quad \sigma_{3}=0,
$$

and the equations of $(G)$ are

$$
z=\rho_{1}+\sigma_{1} \sin 2 \phi, \quad t=\sigma_{1} \cos 2 \phi .
$$

The fundamental equations (3.6) become

$$
C-K=2 \sigma_{1}^{2}-\stackrel{2}{\rho_{1}}
$$

$$
\begin{gathered}
\delta_{2} \rho_{1}-\delta_{1} \sigma_{1}-2 \alpha \sigma_{1}+\nu_{2} \sigma_{1}=0, \quad \delta_{1} \rho_{1}-\delta_{2} \sigma_{1}-2 \beta \sigma_{1}-\nu_{1} \sigma_{1}=0 \\
\delta_{2} \sigma_{1}+2 \beta \sigma_{1}+\nu_{1} \sigma_{1}-\nu_{2} \rho_{1}=0, \quad-\delta_{1} \sigma_{1}-2 \alpha \sigma_{1}+\nu_{2} \sigma_{1}-\nu_{1} \rho_{1}=0 \\
\delta_{2} \nu_{1}-\delta_{1} \nu_{2}+\beta \nu_{1}-\alpha \nu_{2}-2 \sigma_{1}^{2}=0
\end{gathered}
$$

$\left.{ }^{(10}\right)$ A surface in a Riemannian 3-space $V_{3}$ is called a sphere if its normal curvature in $V_{3}$ is constant for every direction at every point. 
If $\rho_{1}=0$, we have the case of an $R$-surface. If $\rho_{1} \neq 0$, we have on adding $(4.54)_{2,3}$ and substracting $(4.54)_{1,4}$,

$$
\nu_{1}=-\delta_{2} \log \rho_{1}, \quad \nu_{2}=\delta_{1} \log \rho_{1},
$$

respectively. Substitution of these values for $\nu_{1}, \nu_{2}$ in (4.55) gives

$$
-\left(\delta_{1} \delta_{1}+\delta_{2} \delta_{2}+\beta \delta_{2}+\alpha \delta_{1}\right) \log \rho_{1}-2 \sigma_{1}^{2}=0,
$$

that is

$$
\Delta_{2} \log \rho_{1}+2 \sigma_{1}^{2}=0 .
$$

From this it follows that if $\rho_{1}=$ const. $\neq 0, \sigma_{1}$ must be zero. This, together with (4.52), shows that the $V_{2}$ is a sphere in a plane $S_{3}$ in $S_{4}$ (cf. SchoutenStruik [15, pp. 99-101, 148-150]). Our theorem is thus proved.

Next we prove the following theorem.

THEOREM 4.14. Among the surfaces $V_{2}$ in $S_{4}$ whose curvature conic $(G)$ at each point $P$ has a focus at $P$, the one for which $(G)$ is of constant shape is an $R$-surface. $A V_{2}$ can be imbedded in an $S_{4}$ such that the curvature conic at each point $P$ has a focus at $P$ without making $V_{2}$ an $R$-surface, if and only if the linear element of $V_{2}$ is reducible to the form

$$
d s^{2}=d u^{2} / \operatorname{ch}^{2} \psi+d v^{2} / \operatorname{sh}^{2} \psi,
$$

where $\psi$ satisfies a certain partial differential equation of the fourth order in the independent variables $u, v$.

Proof. From $(3.7)_{3}$ we can always choose $i_{(3)}^{\kappa}, i_{(1)}^{a}$ so that

$$
\rho_{3}=\sigma_{1}=\sigma_{2}=0, \quad \sigma_{3}^{2}=-\rho_{1} \rho_{2} \neq 0 .
$$

We have an $R$-surface if and only if $\rho_{2}=-\rho_{1}$. The fundamental equations (3.6) become

$$
\begin{gathered}
C-K=2 \sigma_{3}^{2} \\
\delta_{2 \rho_{1}}+\beta\left(\rho_{1}-\rho_{2}\right)-\nu_{1} \sigma_{3}=0, \quad \delta_{1} \rho_{2}-\alpha\left(\rho_{1}-\rho_{2}\right)-\nu_{2} \sigma_{3}=0 \\
\delta_{1} \sigma_{3}+2 \alpha \sigma_{3}+\nu_{2} \rho_{1}=0, \quad \delta_{2} \sigma_{3}+2 \beta \sigma_{3}+\nu_{1} \rho_{2}=0 \\
\delta_{2} \nu_{1}-\delta_{1} \nu_{2}+\beta \nu_{1}-\alpha \nu_{2}+\left(\rho_{1}-\rho_{2}\right) \sigma_{3}=0 .
\end{gathered}
$$

Multiply $(4.61)_{1,4}$ by $\rho_{2}, \sigma_{3}$ respectively, and add. The result is

$$
\rho_{2} \delta_{2} \rho_{1}+\beta \rho_{2}\left(\rho_{1}-\rho_{2}\right)+2^{-1} \delta_{2} \sigma_{3}^{2}+2 \beta \sigma_{3}^{2}=0 .
$$

Using here the value for $\sigma_{3}$ from (4.59), we have after simpiification

$$
2^{-1} \delta_{2} \log \frac{\rho_{1}}{\rho_{2}}-\beta\left(\frac{\rho_{2}}{\rho_{1}}+1\right)=0 .
$$


Similarly from $(4.61)_{2,8}$ we can prove that

$$
2^{-1} \delta_{1} \log \frac{\rho_{2}}{\rho_{1}}-\alpha\left(\frac{\rho_{1}}{\rho_{2}}+1\right)=0 .
$$

If $(G)$ is of constant shape,

$$
\rho_{2} / \rho_{1}=\text { const. }=-A \neq 0 .
$$

Then (4.63) become

$$
\beta\left(\rho_{2} / \rho_{1}+1\right)=0, \quad \alpha\left(\rho_{1} / \rho_{2}+1\right)=0 .
$$

Therefore either $\rho_{1}+\rho_{2}=0$ so that $V_{2}$ is an $R$-surface, or $\alpha=\beta=0$. For the latter case, Liouville's formula (1.6) tells us that $K=0$. Therefore it follows from (4.59)-(4.61) that

$$
A \rho_{1}^{2}=2 \sigma_{3}^{2}=C=\text { const., } \quad \nu_{1}=\nu_{2}=0 .
$$

These, however, contradict (4.62). Hence the $R$-surface is the only $V_{2}$ of this type for which $(G)$ is of constant shape.

Now excluding the case of an $R$-surface, we can put

$$
\rho_{1} / \rho_{2}=-\operatorname{th}^{2} \psi \quad(\psi \neq \text { const. }) .
$$

Then equations (4.63) can be reduced to

$$
\delta_{1} \psi=-\alpha \text { th } \psi, \quad \delta_{2} \psi=-\beta \operatorname{coth} \psi .
$$

Taking $i_{(1)}^{a}, i_{(2)}^{a}$ as parametric curves, we have

$$
d s^{2}=E d u^{2}+G d v^{2},
$$

and equations (4.66) are equivalent to (cf. (1.13))

$$
\partial_{u} \log \left(G^{1 / 2} \operatorname{sh} \psi\right)=0, \quad \partial_{v} \log \left(E^{1 / 2} \operatorname{ch} \psi\right)=0,
$$

which give

$$
G \operatorname{sh}^{2} \psi=1, \quad E \operatorname{ch}^{2} \psi=1,
$$

after an obvious change of $u, v$. Hence (4.58) is proved.

From (4.59) and (4.65) it follows that

$$
\rho_{1}=\sigma_{3} \text { th } \psi, \quad \rho_{2}=-\sigma_{3} \operatorname{coth} \psi .
$$

Putting these in $(4.61)_{3,4}$ and solving for $\nu_{1}, \nu_{2}$ we have

$$
\nu_{1}=\left(\delta_{2} \log \sigma_{3}+2 \beta\right) \text { th } \psi, \quad \nu_{2}=-\left(\delta_{1} \log \sigma_{3}+2 \alpha\right) \operatorname{coth} \psi .
$$

Now substitute in (4.62) these values for $\nu_{1}$ and $\nu_{2}$, the values for $\alpha, \beta$ from (4.66), and finally the value for $\sigma_{3}$ from (4.59), where $K$ is to be calculated from (4.58) and is therefore a differential expression of the second order in $\psi$. Then the result is a differential equation of the fourth order in $\psi$ with inde- 
pendent variables $u, v$. That this differential equation be satisfied is evidently a necessary and sufficient condition for the $V_{2}$ with linear element (4.58) to be imbeddable in $S_{4}$ in the manner required in Theorem 4.14 . Hence the theorem is proved.

Let us remark that the Kommerell conic of this type of $V_{2}$ is always a circle (see end of $\$ 2.3$ ), and that a detailed discussion of this type of $V_{2}$ in an $R_{4}$ can be found in Calapso's papers [3,4].

5.1. Ruled surface in $S_{4}$ (cf. Coburn [5]). By definition a ruled surface in $S_{4}$ is a $V_{2}$ which contains a congruence of geodesics of $S_{4}$. In particular, it is a developable if the geodesics of this congruence envelope a curve in $V_{2}$. A ruled $V_{2}$ which is not a developable will be called a skew ruled $V_{2}$. It is well known that a geodesic of a Riemannian $V_{n}$ is also a geodesic of any subspace in which it lies, and that the normal curvature vector of the subspace in $V_{n}$ for the direction of the geodesic is zero. Therefore the curvature conic $(G)$ at each point $P$ of a ruled $V_{2}$ in $S_{4}$ passes through $P$, the point $P$ on $(G)$ being the end point of the zero normal curvature vector of $V_{2}$ for the geodesic of the congruence at $P$.

As we saw in $(3.7)_{4}$, we can choose $i_{(p)}^{\kappa}, i_{(c)}^{a}$ for a ruled $V_{2}$ in $S_{4}$ so that

$$
\begin{gathered}
\rho_{1}=\sigma_{1}=\sigma_{3}=0, \\
\beta=0 .
\end{gathered}
$$

We know (Schouten-Struik [15, pp. 99-101, 148-150]) that a surface in an $S_{4}$ is a developable if and only if the curvature $H_{c b}^{\cdot * *}$ is of rank 1 . Therefore it follows from (5.1) that a ruled $V_{2}$ in $S_{4}$ is a developable if and only if $\rho_{3}=0$.

Let us first study the skew ruled $V_{2}$ in $S_{4}$. Equations $(3.6)_{1}$ is

$$
C-K=\stackrel{2}{\rho_{3}} \text {. }
$$

Equation (3.6) is $\nu_{1} \rho_{3}=0$, which gives, since $\rho_{3} \neq 0$,

$$
\nu_{1}=0 \text {. }
$$

Then equation (3.6) $)_{2}$ becomes

$$
\delta_{1} \rho_{3}+2 \alpha \rho_{3}=0 .
$$

Substitution of the value for $\rho_{3}$ from (5.3) gives

$$
\delta_{1} \log (C-K)+4 \alpha=0 .
$$

Equation $(3.6)_{3}$ is

$$
\delta_{1} \rho_{2}+\alpha \rho_{2}-\delta_{2} \rho_{3}=0 .
$$

Solving equation (3.6) ${ }_{5}$ for $\nu_{2}$, we have

$$
\nu_{2}=-\frac{1}{\rho_{3}}\left(\delta_{1} \sigma_{2}+\alpha \sigma_{2}\right),
$$


which, because of (5.5), can be written

$$
\nu_{2}=-\delta_{1} \lambda+\alpha \lambda \text {, where } \lambda=\sigma_{2} / \rho_{3} .
$$

Substituting this in the last equation of (3.6), we have

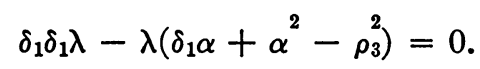

But since $\beta=0$, equation (1.6) reduces to $K=-\left(\delta_{1} \alpha+\alpha^{2}\right)$. On account of this and (5.3), the preceding equation simplifies into

$$
\delta_{1} \delta_{1} \lambda=C \lambda \text {. }
$$

Equations (5.1)-(5.8) are the fundamental equations for a skew ruled $V_{2}$ in $S_{4}$. Equation $\left(5.5^{\prime}\right)$ involves only $K$ and the congruences $i_{(1)}^{a}, i_{(2)}^{a}$. Let this be satisfied by a $V_{2}$ and a geodesic congruence $i_{(1)}^{a}$ of it. Then when $V_{2}$ is referred to $i_{(1)}^{a}, i_{(2)}^{a}$ as parametric curves $v=$ const. and $u=$ const., equations (5.7) and (5.8) determine $\rho_{2}$ and $\lambda$ to within one and two arbitrary functions of $v$, respectively. And for any $\lambda$ thus determined, equation (5.7) gives a unique value for $\nu_{2}$. Therefore $\left(5.5^{\prime}\right)$ is a necessary and sufficient condition for a given $V_{2}$ with a given geodesic congruence $i_{(1)}^{a}$ to be imbeddable in $S_{4}$ as a skew ruled $V_{2}$ with $i_{(1)}^{a}$ as rulings. Moreover, with $\rho_{3} \neq 0$ and given $i_{(1)}^{a}$, equations (5.1) will determine $i_{(p)}^{\kappa}$ to within signs. Hence the arbitrariness of the imbedding is equal to the arbitrariness of the solution for the scalars $\rho$ 's, $\sigma$ 's and $\nu$ 's. It is seen further that if we put $\lambda=0$, the equations (5.1)-(5.8) reduce to a consistent system of equations; in fact, they reduce to the fundamental equations for a ruled $V_{2}$ in an $S_{3}$ of constant scalar curvature $C / 3$ with second fundamental tensors $h_{c b}=\rho_{1} i_{c}^{(1)} i_{b}^{(1)}+\rho_{2} i_{c}^{(2)} i_{b}^{(2)}+\rho_{3}\left(i_{c}^{(1)} i_{b}^{(2)}+i_{c}^{(2)} i_{b}^{(1)}\right)$ (cf. (7.2) below). Hence we have the following theorem.

TheOREM 5.1. Given on a $V_{2}$ a geodesic congruence $i_{(1)}^{a}, V_{2}$ can be imbedded in an $S_{4}$ of scalar curvature $C$ as a skew ruled surface with $i_{(1)}^{a}$ as rulings if and only if $\left(5.5^{\prime}\right)$ is satisfied. If this condition is satisfied, the imbedding, to within a motion in $S_{4}$, depends on three arbitrary functions of a single variable. A skew ruled $V_{2}$ in $S_{4}$ can be deformed into a ruled $V_{2}$ in an $S_{3}$ of scalar curvature equal to that of $S_{4}$ such that rulings become rulings and that one of the second fundamental tensors of $V_{2}$ in $S_{4}$ is identical with the second fundamental tensor of $V_{2}$ in $S_{3}$ (cf. Theorem 7.1).

5.2. Certain pair of applicable skew ruled $V_{2}$ in $S_{4}$. Let $V_{2}, \bar{V}_{2}$ be two applicable skew ruled surfaces in $S_{4}$ such that rulings correspond to rulings and the curvature conics of $V_{2}, \bar{V}_{2}$ at corresponding points are equal. We suppose that $i_{(p)}^{\kappa}, i_{(c)}^{a}, \bar{i}_{(p)}^{\kappa}, \bar{i}_{(c)}^{a}$ have been so chosen that equations (5.1)-(5.8), $\left(5.1^{-}\right)-\left(5.8^{-}\right)$hold. Here and in what follows we use a dash to indicate quantities and equations belonging to $\bar{V}_{2}$. Since $d \bar{s}^{2}=d s^{2}$ and rulings correspond, the vectors $\bar{i}_{(c)}^{a}, i_{(c)}^{a}$ differ at most by signs, and therefore we may readjust the senses of $\bar{i}_{(c)}^{a}$ so that $i_{(c)}^{a}=i_{(c)}^{a}$. Then 


$$
\bar{K}=K, \quad \bar{\delta}_{c}=\delta_{c}, \quad \bar{\alpha}=\alpha, \quad \bar{\beta}=\beta=0 .
$$

Now the curvature conics $(G),(\bar{G})$ of $V_{2}, \bar{V}_{2}$ at corresponding points are equal if and only if

$$
\bar{\sigma}_{2} \bar{\rho}_{3}= \pm \sigma_{2} \rho_{3}, \quad \bar{\rho}_{2}^{2}+\bar{\sigma}_{2}^{2}+4 \bar{\rho}_{3}^{2}=\stackrel{\rho}{2}_{2}^{2}+\sigma_{2}^{2}+4 \rho_{3}^{2}
$$

are satisfied. These two equations express the fact that $(G),(\bar{G})$ have equal areas and director circles (cf. (2.22), (2.23)).

Equations $\left(5.1^{-}\right)$determine $\bar{i}_{(p)}^{k}$ to within signs. Equations (5.3), (5.3-) and (5.9) give $\bar{\rho}_{3}= \pm \rho_{3}$, from which and (5.10) we see that the senses of $i_{(p)}^{k}$ can be so adjusted that

$$
\bar{\rho}_{3}=\rho_{3}, \quad \bar{\sigma}_{2}=\sigma_{2}, \quad \bar{\rho}_{2}= \pm \rho_{2} .
$$

If $\bar{\rho}_{2}=\rho_{2}$, it follows from (5.11), (5.1)-(5.8) that the scalars $\rho^{\prime}$ 's, $\sigma$ 's, $\nu$ 's are equal for $V_{2}$ and $\bar{V}_{2}$, and consequently $V_{2}$ and $\bar{V}_{2}$ are congruent.

If $\bar{\rho}_{2}=-\rho_{2},(5.11)$ and (3.7) 4 show that the normal curvature of $V_{2}$ at $P$ for the direction $\phi$ is equal to that of $\bar{V}_{2}$ at $\bar{P}$ for the direction $\phi=-\phi$. In this case it follows from (5.9), (5.11), (5.6) and (5.6-) that equation (5.6) breaks up into the two equations

$$
\delta_{2} \rho_{3}=0, \quad \delta_{1} \rho_{2}+\alpha \rho_{2}=.0 .
$$

On account of (5.3), equation (5.12) ${ }_{1}$ is the condition that the congruence of curves $K=$ const. is identical with $i_{(2)}^{a}$, that is, it is the orthogonal congruence to the rulings. Moreover, the integrability condition of (5.12) and (5.5) is $\delta_{2} \alpha=0$, showing that each curve $K=$ const. has constant geodesic curvature.

It is readily seen from (5.6) that equations (5.12) hold for a skew ruled $V_{2}$ in $S_{4}$ if and only if $\rho_{2}=0$ is permissible as part of a solution for (5.1)-(5.8). Now if we put $\rho_{2}=\lambda=0$ in $(5.1)-(5.8)$, the latter equations reduce to the fundamental equations of a minimal ruled $V_{2}$ in an $S_{3}$ (in $S_{4}$ ) whose scalar curvature is equal to twice that of $S_{4}$; this can be seen from (7.2). Hence for a skew ruled $V_{2}$ in $S_{4}$, equations (5.12) hold if and only if $V_{2}$ is deformable into a minimal ruled $V_{2}$ in an $S_{3}$ such that rulings correspond to rulings.

Summing up the preceding results we have the following theorems.

THEOREM 5.2. On a skew ruled $V_{2}$ in $S_{4}$, the curves along which the Gaussian curvature $K$ is constant are orthogonal to the rulings if and only if $V_{2}$ is deformable into a minimal ruled $V_{2}$ in an $S_{3}$ such that rulings become rulings. In such a ruled $V_{2}$ each of the curves $K=$ const. has constant geodesic curvature.

THEOREM 5.3. Let $V_{2}, \bar{V}_{2}$ be two applicable skew ruled surfaces in an $S_{4}$ with rulings corresponding to rulings and equal curvature conics at corresponding points. If $V_{2}$ is not deformable into a minimal ruled surface in an $S_{3}$ such that rulings become rulings, then $V_{2}$ and $\bar{V}_{2}$ are congruent. If $V_{2}$ is deformable in the manner just described, $V_{2}$ and $\bar{V}_{2}$ may or may not be congruent. If they are not then the normal curvature of $V_{2}$ at any point $P$ for any direction $i^{a}$ is equal to that 
of $\bar{V}_{2}$ at the corresponding point $\bar{P}$ for the direction whose reflection with respect to the ruling of $\bar{V}_{2}$ at $\bar{P}$ corresponds to $i^{a}$.

When $P$ is a vertex of $(G), \rho_{2}=0$ and $\lambda$ determines the shape of $(G)$, as is seen from figure 4. It follows from (5.8) that $\lambda=$ const. $\neq 0$ is permissible as part of a solution to (5.1)-(5.8) when and only when $C=0$. Hence we have the following theorem.

THEOREM 5.4. In an $S_{4}$ there exists a skew ruled surface whose curvature conic at each point $P$ is a nondegenerate ellipse of constant shape having a vertex at $P$ if and only if $S_{4}$ is an $R_{4}$.

5.3. The linear element of a skew ruled $V_{2}$ in $S_{4}$. This linear element can be found from $\left(5.5^{\prime}\right)$. Referring to $i_{(c)}^{a}$ as parametric curves, we have

$$
d s^{2}=d u^{2}+G d v^{2},
$$

$v=$ const. being the geodesic congruence $i_{(1)}^{a}$. For (5.11),

(5.14) $\delta_{1}=\partial_{u}, \delta_{2}=\frac{\partial_{v}}{G^{1 / 2}}, \alpha=\partial_{u} \log G^{1 / 2}, \beta=0 . K=-\frac{1}{G^{1 / 2}} \partial_{u} \partial_{u} G^{1 / 2}$

Equation (5.5') is

$$
\partial_{u} \log (C-K)+2 \partial_{u} \log G=0
$$

that is,

$$
(C-K) G^{2}=-V_{1},
$$

where and throughout this section $V_{1}, V_{2}\left({ }^{11}\right)$, and so on, denote arbitrary functions of $v$ alone. Using the value for $K$ from (5.12), this becomes

$$
C G^{2}+\left(G^{1 / 2}\right)^{3} \partial_{u} \partial_{u} G^{1 / 2}=-V_{1}
$$

integration of which gives

$$
C G+\left(\partial_{u} G^{1 / 2}\right)^{2}=V_{2}+V_{1} / G
$$

that is,

$$
\left(\partial_{u} G\right)^{2}=4\left(-C G^{2}+V_{2} G+V_{1}\right) .
$$

In particular, when $C=0$, we have from this

$$
G=V_{2} u^{2}+2 V_{3} u+V_{4} .
$$

From (5.3) and (5.12) $)_{1}$, if the congruence $K=$ const. is orthogonal to the rulings, $K$ is a function of $u$ alone. This is the case if and only if $G=U(u) V(v)$, as is seen from (5.15) and (5.14). Hence we have the following theorem.

(11) See footnote 9. 
TheOREM 5.5. $A V_{2}$ can be imbedded in an $S_{4}$ as a skew ruled surface with $v=$ const. as rulings if and only if its linear element is reducible to the form (5.13) in which $G$ satisfies (5.16). The congruence $K=$ const. is orthogonal to the rulings if and only if $G$, as given by (5.16), is a product of a function of $u$ alone by a function of $v$ alone.

5.4. Developable in $S_{4}$. The curvature conic at each point $P$ of a developable $V_{2}$ in $S_{4}$ is a line segment $(G)$ ending at $P$, and the congruence along which $V_{2}$ has zero normal curvature in $S_{4}$ is geodesic in $V_{2}$. Therefore by choosing $i_{(3)}^{\kappa}$ along $(G)$ and $i_{(1)}^{a}$ as the geodesic congruence, we have

$$
\rho_{1}=\rho_{3}=\sigma_{1}=\sigma_{2}=\sigma_{3}=0, \quad \beta=0 .
$$

Here $\rho_{2}$ is or is not equal to zero according as $V_{2}$ is or is not plane in $S_{4}$.

If $\rho_{2} \neq 0$ and $i_{(1)}^{a}$ is given, (5.16) will determine $i_{(p)}^{\kappa}$ to within signs. For this case the fundamental equations (3.6) become

$$
\begin{gathered}
C-K=0, \\
\delta_{1} \rho_{2}+\alpha \rho_{2}=0, \quad \nu_{1}=0, \quad \delta_{1} \nu_{2}+\alpha \nu_{2}=0 .
\end{gathered}
$$

Equation (5.19) shows that $V_{2}$ has constant Gaussian curvature. Referring $V_{2}$ to $i_{(1)}^{a}, i_{(2)}^{a}$ as parametric curves $v=$ const., $u=$ const., we see that $\rho_{2}, \nu_{2}$ are determined by $(5.20)_{1,3}$, each to within an arbitrary function of $v$. Furthermore, we note that $\rho_{2}=\nu_{1}=\nu_{2}=0$ is a particular solution of (5.20).

For a plane $V_{2}$ in $S_{4}$, all the $\rho$ 's and $\sigma$ 's are zero, and the fundamental equations (3.6) reduce to (5.19) and

$$
\nu_{1}=\text { arbitrary, } \quad \delta_{1} \nu_{2}+\alpha \nu_{2}=0 .
$$

Hence summing up the preceding results we have the following theorem.

Theorem 5.6. $A V_{2}$ can be imbedded as a developable in an $S_{4}$ of scalar curvature $C$ if and only if it has constant Gaussian curvature $C$. Given arbitrarily a curve $(C)$ in such $V_{2}$, it is possible to imbed $V_{2}$ in $S_{4}$ as a developable with the geodesics tangent to $(C)$ as rulings. The imbedding depends, to within a motion in $S_{4}$, on two arbitrary functions, one of one variable and the other of two variables, or both of two variables, according as the developable is or is not plane. A developable in $S_{4}$ can be deformed into a plane $V_{2}$ in an $S_{3}$ whose scalar curvature is equal to twice that of $S_{4}$.

It is well known that an $R_{2}$ (that is, a $V_{2}$ with $K=0$ ) in an $R_{3}$ is a developable. This is no longer the case for an $R_{2}$ in $R_{4}$; for, the curvature tensor $H_{c b}^{\cdot *}$ of an $R_{2}$ in $R_{4}$ is not necessarily of rank 1 (cf. Theorem 2.2). But for a ruled $R_{2}$ in an $R_{4}$ we have $K=C=0$, so that $\rho_{3}=0$ is a consequence of (5.1) and (3.6) $)_{1}$. Hence we have the føllowing theorem.

Theorem 5.7. A ruled' $R_{2}$ in an $R_{4}$ is a developable. 
Let $V_{2}$ and $\bar{V}_{2}$ be two non-plane developables in $S_{4}$ which are applicable to each other such that rulings correspond to rulings and the curvature conics (which are line segments) at corresponding points are equal. Then as in $\$ 5.2$, we have (5.9) and $\bar{\rho}_{2}=\rho_{2}$. Since $\nu_{2}$ has only to satisfy equation $(5.20)_{3}$ and therefore depends on an arbitrary function of one variable, we have proved the following counterpart to Theorem 5.3:

Theorem 5.8. A non-plane developable in an $S_{4}$ can be deformed into infinitely many developables in $S_{4}$ such that rulings become rulings and the curvature conics at corresponding points are equal. The deformation depends on one arbitrary function of one variable.

6. Surface in $R_{4}$ with an orthogonal net of Voss. A $V_{2}$ in an $R_{4}$ is said to have a net of Voss if its two conjugate congruences are uniquely determined and the curves of the two congruences are geodesics of $V_{2}$. A few properties of a $V_{2}$ in $R_{4}$ with an orthogonal net of Voss were given by Zitto [17] (cf. also Schouten-Struik [15, pp. 150-153]). Here we obtain the finite equations of such a $V_{2}$ by actually integrating its fundamental equations.

Since the conjugate net is unique and orthogonal, the curvature conic $(G)$ at each point $P$ is a line segment not passing through $P$ (see paragraph below (2.29)). And since $V_{2}$ has mutually orthogonal congruences of geodesics, it follows from Liouville's formula (1.6) that $K=0$, that is, the $V_{2}$ is an $R_{2}$. Consequently, the line segment $(G)$ at each point $P$ of $V_{2}$ subtends a right angle at $P$ (cf. Theorem 2.2).

Now if we choose $i_{(p)}^{\kappa}$ suitably and take $i_{(c)}^{a}$ as the unit tangent vectors to the net, we have, as in (3.7),

$$
\rho_{1}=\rho_{3}=\sigma_{2}=\sigma_{3}=0 .
$$

Since $i_{(c)}^{a}$ are geodesic congruences,

$$
\alpha=\beta=0 .
$$

Furthermore, since the line segment $(G)$ does not pass through $P$, neither $\rho_{2}$ nor $\sigma_{1}$ can be zero. Then the fundamental equations (3.6) reduce to

$$
\delta_{1} \rho_{2}=\delta_{2} \sigma_{1}=0, \quad \nu_{1}=\nu_{2}=0 .
$$

For an $R_{2}$, equations (6.1)-(6.3) have solutions for $i_{(c)}^{a}$ and the $\rho$ 's, $\sigma$ 's, $\nu$ 's. Therefore, there exist in an $R_{4}$ surfaces with an orthogonal net of Voss.

We now proceed to find the finite equations of such $V_{2}$ 's. With $i_{(c)}^{a}$ as parametric curves, the linear element of $R_{2}$ is

$$
d s^{2}=d u^{2}+d v^{2},
$$

so that $\Gamma_{c b}^{a}=0$, and

$$
\stackrel{a}{i_{(1)}}=i_{a}^{(1)}=(1,0), \quad \underset{i_{(2)}}{i}=i_{a}^{(2)}=(0,1), \quad \delta_{1}=\partial_{u}, \quad \delta_{2}=\partial_{v} .
$$


Then it follows from (6.1)-(6.3) that

$$
h_{c b}^{(3)}=V^{0} i_{c}^{(2)} i_{b}^{(2)}, \quad h_{c b}^{(4)}=U^{0} i_{c}^{(1)} i_{b}^{(1)}, \quad v_{c}=0,
$$

where $U^{0}\left(=\sigma_{1}\right), V^{0}\left(=\rho_{2}\right)$ are respectively nonzero functions of $u, v$ alone. Therefore the fundamental equations (3.1) become

$$
\begin{aligned}
\delta_{c} y^{k} & =B_{c}^{k}, & \delta_{c} B_{b}^{k} & =V^{0} i_{c}{ }^{(2)} i_{(2)}{ }^{(2)} i_{(3)}^{k}+U^{0} i_{c}^{(1)} i_{b}^{(1)} i_{(4)}^{k}, \\
\delta_{c} i_{(3)}^{k} & =-V^{0} i_{c}{ }^{(2)} i_{(2)}^{b} B_{b}^{k}, & \delta_{c} i_{(4)}^{k} & =-U^{0} i_{c}^{(1)} i_{(1)}^{b} B_{b}^{k},
\end{aligned}
$$

where $y^{\wedge}$ are rectangular Cartesian coordinates in $R_{\mathbf{4}}$.

We shall now solve this system of equations. Equations $(6.7)_{3.4}$ for $c=1,2$, respectively, are $\partial_{u} i_{(3)}^{\kappa}=0, \partial_{v} i_{(4)}^{\kappa}=0$. Therefore

$$
i_{(3)}^{*}=V^{*}, \quad i_{(4)}^{*}=U^{*},
$$

where $V^{*}, U^{*}$ are vectors in $R_{4}$ depending on $v, u$ alone, respectively. Then $(6.7)_{3,4}$ for $c=2,1$, respectively, are

$$
\left(V^{*}\right)^{\prime}=-V^{0} B_{2}^{*}, \quad\left(U^{*}\right)^{\prime}=-U^{0} B_{1}^{*} .
$$

These together with $(6.7)_{1}$ give

$$
-y^{*}=\int \frac{\left(U^{k}\right)^{\prime}}{U^{0}} d u+\int \frac{\left(V^{*}\right)^{\prime}}{V^{0}} d v .
$$

Substituting in $(6.7)_{2}$ the values for $i_{(3)}^{\kappa}, i_{(4)}^{\kappa}$, and $B_{c}^{\kappa}$ from (6.8) and (6.9) we have

$$
\left(\left(U^{*}\right)^{\prime} / U^{0}\right)^{\prime}=-U^{0} U^{*}, \quad\left(\left(V^{*}\right)^{\prime} / V^{0}\right)^{\prime}=-V^{0} V^{*} .
$$

If we multiply the first equation by $2\left(U^{*}\right)^{\prime} / U^{0}$ and integrate, the result is

$$
\left(\left(U^{*}\right)^{\prime} / U^{0}\right)^{2}+\left(U^{*}\right)^{2}=\text { const. } \equiv\left(A^{*}\right)^{2} .
$$

Therefore we may put

$$
(6.12)_{1} \quad U^{*}=A^{*} \cos \theta^{*}, \quad\left(U^{*}\right)^{\prime} / U^{0}=A^{*} \sin \theta^{*} \text {. }
$$

To determine $\theta$ we need only substitute the first equation in the second one and find that $\left(\theta^{*}\right)^{\prime}=-U^{0}$. Hence

$$
\theta^{*}=-\int U^{0} d u+C^{k} \quad\left(C^{k}=\text { const. }\right)
$$

Similarly, we derive from $(6.11)_{2}$

(6.13) $V^{*}=B^{*} \cos \phi^{*}, \quad\left(V^{*}\right)^{\prime} / V^{0}=B^{*} \sin \phi^{*}, \quad \phi^{*}=-\int V^{0} d v+D^{\alpha}$ ( $B^{*}, D^{k}$ const.). 
Using (6.12) and (6.13) in (6.10), we have

$$
\begin{aligned}
-y^{*}= & A^{*} \int \sin \left(-\int U^{0} d u+C^{*}\right) d u \\
& +B^{*} \int \sin \left(-\int V^{0} d v+D^{*}\right) d v .
\end{aligned}
$$

Suppose that suitable constants of integration have been added to

$$
\int U^{0} d u \equiv-U, \quad \int V^{0} d v \equiv-V
$$

so that $U=0, V=0$ at some point $P_{0}\left(u_{0}, v_{0}\right)$. Then at $P_{0}$ we have

$$
\left(\theta^{*}\right)_{0}=C^{*}, \quad\left(\phi^{*}\right)_{0}=D^{*},
$$

$$
\begin{array}{rlrl}
\left(i_{(3)}^{*}\right)_{0} & =\left(V^{*}\right)_{0}=B^{*} \cos D^{*}, & \left(i_{(4)}^{*}\right)_{0} & =\left(U^{*}\right)_{0}=A^{*} \cos C^{*}, \\
\left(B_{1}^{*}\right)_{0} & =-A^{*} \sin C^{*}, & \left(B_{2}^{*}\right)_{0}=-B^{*} \sin D^{*} .
\end{array}
$$

Now according to Eisenhart's theorem quoted in $\$ 3$, the vectors $\left(B_{1}^{*}\right)_{0}$, $\left(B_{2}^{k}\right)_{0},\left(i_{(3)}^{\kappa}\right)_{0},\left(i_{(4)}^{\kappa}\right)_{0}$ can be chosen arbitrarily as long as they satisfy the following relations

$$
\sum_{\alpha} B_{c}^{\alpha} B_{b}^{\alpha}=\delta_{c b}, \quad B_{i}^{\alpha} i_{k}^{(p)}=0, \quad i_{(p)}^{\alpha} i_{k}^{(q)}=\delta_{p}^{q} \quad(b, c=1,2 ; p, q=3,4) .
$$

Therefore we may choose $-A^{*} \sin C^{\kappa}=\left(B_{1}^{*}\right)_{0}=(0,1,0,0),-B^{\kappa} \sin D^{*}=\left(B_{2}^{\kappa}\right)_{0}$ $=(1,0,0,0), B^{x} \cos D^{x}=\left(i_{(3)}^{\kappa}\right)_{0}=(0,0,-1,0), A^{x} \cos C^{k}=\left(i_{(4)}^{\kappa}\right)_{0}=(0,0,0,-1)$; that is,

$$
\begin{aligned}
A^{1}=A^{8} & =0, & B^{2}=B^{4} & =0, \\
A^{2} \sin C^{2} & =-1, & & A^{4} \sin C^{4}=0, \\
A^{2} \cos C^{2} & =0, & A^{4} \cos C^{4} & =-1, \\
B^{1} \sin D^{1} & =-1, & B^{3} \sin D^{8} & =0, \\
B^{1} \cos D^{1} & =0, & B^{3} \cos D^{8} & =-1 .
\end{aligned}
$$

A solution of these equations is

$$
\begin{array}{ll}
A^{*}=(0,-1,0,-1), & B^{k}=(-1,0,-1,0), \\
C^{2}=2^{-1} \pi, \quad C^{4}=0, & D^{1}=2^{-1} \pi, \quad D^{3}=0 .
\end{array}
$$

If we use these in (6.15) and simplify, the result is

(6.20) $y^{k}=\left(\int \cos V d v, \int \cos U d u, \int \sin V d v, \int \sin U d u\right)$,

where $U, V$ are as defined by (6.15) and are therefore arbitrary nonconstant 
functions of $u, v$, respectively. Other solutions of (6.18) lead to the same result (6.20). Hence we have the following theorem.

THEOREM 5.1. Any surface in $R_{4}$ with an orthogonal net of Voss is congruent to one of the surfaces (6.20), where $y^{*}$ are rectangular Cartesian coordinates in $R_{4}$ and $U, V$, are respectively nonconstant functions of $u, v$ alone.

The simplest of such surfaces is

$$
y^{\alpha}=(\sin v, \sin u,-\cos v,-\cos u),
$$

which is obtained by putting $V=v, U=u$ in (6.20). This surface is well known for the fact that it is a closed $R_{2}$ in $R_{4}$ (cf. Tompkins [16]).

7. A correspondence between a $V_{2}$ in an $S_{3}$ and an isometric $V_{2}$ in an $S_{4}$. We propose to solve the following problem: Given $a V_{2}$ in an $S_{3}$, to find the imbedding of an isometric $V_{2}$ in an $S_{4}$ such that one of the second fundamental tensors of $V_{2}$ in $S_{4}$ is identical with the second fundamental tensor of $V_{2}$ in $S_{3}$.

N. Coburn [5] gave two examples of such a correspondence, one for an arbitrary $V_{2}$ in $S_{3}$ and one for a ruled $V_{2}$ in $S_{3}$. Here we give a complete solution of the problem by proving the following theorem.

THEOREM 7.1. Let the constant scalar curvatures of an $S_{3}$ and an $S_{4}$ be $C^{\prime}$ and $C$, respectively. Then our problem has the following, and only the following, solutions (where the imbedding is described to within a motion in $S_{4}$ ):

(1a) $C<C^{\prime}$. For an arbitrarily given $V_{2}$ in $S_{3}$, there is in general one and only one solution. The curvature conic of the isometric $V_{2}$ in $S_{4}$ at each point $P$ of this $V_{2}$ is a line segment at the constant distance $\left(C^{\prime}-C\right)^{1 / 2}$ from $P$, and the imbedding of this $V_{2}$ in $S_{4}$ is unique.

(1b) $C \neq C^{\prime}$. If the linear element of $V_{2}$ in $S_{3}$ is reducible to

$$
d s^{2}=\frac{\epsilon_{1} d u^{2}+\epsilon_{2} e^{2 \theta} d v^{2}}{1-\epsilon e^{2 \theta}}\left(\theta \neq \text { const. } ; \epsilon, \epsilon_{1}, \epsilon_{2}= \pm 1\right)
$$

when referred to its lines of curvature, there is another solution than (1a). The imbedding of this isometric $V_{2}$ in $S_{4}$ is unique or depends on one arbitrary constant according as the lines of curvature of $V_{2}$ in $S_{3}$ do not form or form an isometric net, that is, according as $\theta \neq U+V$ or $\theta=U+V$, where $U, V$ are functions of $u, v$ alone. The curvature conic $(G)$ of this $V_{2}$ in $S_{4}$ is always a line segment.

(2) $C=C^{\prime}$. (2a) For any $V_{2}$ in $S_{3}$, there is a trivial solution; the $V_{2}$ in $S_{3}$ may be considered as a surface in $S_{4}$.

(2b) For an arbitrarily given $V_{2}$ in $S_{3}$, there is in general one and only one solution other than the trivial one (1a). The imbedding of this isometric $V_{2}$ in $S_{4}$ depends on an arbitrary function of one variable and on an arbitrary solution of a linear partial differential equation of second order in two independent variables. The curvature conic of this $V_{2}$ in $S_{4}$ is in general not degenerate. 
(2c) If the $V_{2}$ in $S_{3}$ has a geodesic congruence of lines of curvature, there is another solution than (2a) and (2b). The imbedding of this isometric $V_{2}$ in $S_{4}$ depends on an arbitrary function of a single variable, and the curvature conic of this $V_{2}$ in $S_{4}$ is always a line segment.

(2d) If the $V_{2}$ in $S_{3}$ is a ruled surface, there is a solution other than (2a) and (2b). The imbedding of this isometric $V_{2}$ in $S_{4}$ depends on two arbitrary functions of one variable. This $V_{2}$ in $S_{4}$ is also a ruled surface, and its curvature conic is in general not degenerate.

Proof. For a $V_{2}$ in an $S_{3}$, let $K$ be its Gaussian curvature; $i_{(1)}^{a}, i_{(2)}^{a}$ two mutually orthogonal congruences; $\beta, \alpha$ their geodesic curvatures (cf. \$1.2); and

$$
h_{c b}=\rho_{1} i_{c}^{(1)} i_{b}^{(1)}+\rho_{2} i_{c}^{(2)} i_{b}^{(2)}+\rho_{3}\left(i_{c}^{(1)} i_{b}^{(2)}+i_{c}^{(2)} i_{b}^{(1)}\right)
$$

the second fundamental tensor. Then the fundamental equations of $V_{2}$ in $S_{3}$ are

$$
\begin{gathered}
C^{\prime}-K=\rho_{3}^{2}-\rho_{1} \rho_{2}, \\
\delta_{2} \rho_{1}-\delta_{1} \rho_{3}+\beta\left(\rho_{1}-\rho_{2}\right)-2 \alpha \rho_{3}=0, \\
\delta_{1} \rho_{2}-\delta_{2} \rho_{3}-\alpha\left(\rho_{1}-\rho_{2}\right)-2 \beta \rho_{3}=0,
\end{gathered}
$$

where $C^{\prime}=-R^{\prime} / 6$ is the constant scalar curvature of $S_{3}$. We notice that these equations are obtainable from (3.6) by putting $\sigma_{1}=\sigma_{2}=\sigma_{3}=0, \nu_{1}=\nu_{2}=0$, and $C=C^{\prime}$.

Let us suppose that a $V_{2}$, isometric to the $V_{2}$ in $S_{3}$, is imbedded in an $S_{4}$ of scalar curvature $C / 6$ such that its second fundamental tensor $h_{c b}^{(3)}$ in $S_{4}$ is identical with the second fundamental tensor (7.1) of $V_{2}$ in $S_{3}$. Using the same $i_{(1)}^{a}, i_{(2)}^{a}$ for both cases, we have, by comparing (7.2) with (3.6),

$$
\begin{gathered}
C-C^{\prime}=\sigma_{3}^{2}-\sigma_{1} \sigma_{2}, \\
\nu_{1} \sigma_{3}=\nu_{2} \sigma_{1}, \quad \nu_{1} \sigma_{2}=\nu_{2} \sigma_{3}, \\
\delta_{2} \sigma_{1}-\delta_{1} \sigma_{3}+\beta\left(\sigma_{1}-\sigma_{2}\right)-2 \alpha \sigma_{3}+\nu_{1} \rho_{3}-\nu_{2} \rho_{1}=0, \\
\delta_{1} \sigma_{2}-\delta_{2} \sigma_{3}-\alpha\left(\sigma_{1}-\sigma_{2}\right)-2 \beta \sigma_{3}+\nu_{2} \rho_{3}-\nu_{1} \rho_{2}=0, \\
\delta_{2} \nu_{1}-\delta_{1} \nu_{2}+\beta \nu_{1}-\alpha \nu_{2}+\left(\rho_{1}-\rho_{2}\right) \sigma_{3}-\left(\sigma_{1}-\sigma_{2}\right) \rho_{3}=0 .
\end{gathered}
$$

Our problem is to solve equations (7.3)-(7.6) for the $\sigma$ 's and $\nu$ 's, the congruences $i_{(1)}^{a}, i_{(2)}^{a}$ and the $\rho$ 's being given and satisfying (7.2). Consider the two cases $C \neq C^{\prime}$ and $C=C^{\prime}$ separately.

Case 1. $C \neq C^{\prime}$. In this case, equations (7.3) and (7.4) are equivalent to

$$
C-C^{\prime}=\sigma_{3}^{2}-\sigma_{1} \sigma_{2} \neq 0, \quad \nu_{1}=\nu_{2}=0 .
$$

Let us choose $i_{(1)}^{a}, i_{(2)}^{a}$ to be the lines of curvature of $V_{2}$ in $S_{3}$, so that

$$
\rho_{3}=0 .
$$


Then equation (7.6) becomes $\left(\rho_{1}-\rho_{2}\right) \sigma_{3}=0$. If $\rho_{2}=\rho_{1}$, our choice of $i_{(1)}^{a}$ is still arbitrary. We may choose it so that $\sigma_{3}=0$. Hence from the preceding equation we always have that

$$
\sigma_{3}=0 \text {. }
$$

Equations (7.8) and (7.9) show that the curvature conic $(G)$ of the $V_{2}$ in $S_{4}$ is a line segment.

On account of (7.7)-(7.9), equations (7.3) and (7.5) become

$$
\begin{gathered}
\sigma_{1} \sigma_{2}=C^{\prime}-C \equiv \epsilon m^{2} \neq 0 \quad(\epsilon= \pm 1, m=\text { const. }), \\
\delta_{2} \sigma_{1}+\beta\left(\sigma_{1}-\sigma_{2}\right)=0, \quad \delta_{1} \sigma_{2}-\alpha\left(\sigma_{1}-\sigma_{2}\right)=0 .
\end{gathered}
$$

In accordance with (7.10), let us put

$$
\sigma_{1}=m e^{\theta}, \quad \sigma_{2}=\epsilon m e^{-\theta} .
$$

Then equations (7.11) become

$$
\delta_{1} \theta=\alpha\left(1-\epsilon e^{2 \theta}\right), \quad \delta_{2} \theta=\beta\left(\epsilon e^{-2 \theta}-1\right) .
$$

Thus we have a desirable imbedding of $V_{2}$ in $S_{4}$ if and only if the lines of curvature $i_{(1)}^{a}, i_{(2)}^{a}$ of $V_{2}$ in $S_{3}$ are such that equations (7.13) admit a solution for $\theta$.

The integrability condition of (7.13) is

$$
\left(1-\epsilon e^{-2 \theta}\right)\left[\left(\delta_{1} \beta-\alpha \beta\right)-e^{2 \theta}\left(\delta_{2} \alpha-\alpha \beta\right)\right]=0 .
$$

This condition can be satisfied in three ways.

Firstly, we may have $1-\epsilon e^{-2 \theta}=0$, that is,

$$
\epsilon=1, \quad \theta=0 \text {. }
$$

Then equations (7.13) are identically satisfied, and equations (7.12) and (7.10) give

$$
\sigma_{1}=\sigma_{2}=m=\text { const. } \quad C^{\prime}-C=m^{2}>0 .
$$

In (7.7), (7.9) and (7.16) we have a solution of equations (7.3)-(7.6), and therefore a desirable imbedding of $V_{2}$ in $S_{4}$. The given $V_{2}$ in $S_{3}$ is entirely unrestricted, but the scalar curvature of $S_{4}$ has to be smaller than twice that of $S_{8}$, as is seen from (7.16). The curvature conic at each point $P$ of such $V_{2}$ in $S_{4}$ is a line segment at a constant distance $\sigma_{1}=m=\left(C^{\prime}-C\right)^{1 / 2}$ from $P$. This proves (1a) of the theorem.

To satisfy (7.14), we may also have

$$
\delta_{2} \alpha-\alpha \beta=0, \quad \delta_{1} \beta-\alpha \beta=0 .
$$

Condition (7.14) being now identically satisfied, equations (7.13) are completely in tegrable. Consequently, $\sigma_{1}$ and $\sigma_{2}$ are uniquely determined when the value of $\theta$ at a certain point of $V$ is given. This is a desirable imbedding of $V_{2}$ 
in $S_{4}$ depending on one arbitrary constant. We note from (7.17) that the lines of curvature $i_{(1)}^{a}, i_{(2)}^{a}$ of $V_{2}$ in $S_{3}$ form an isometric net (cf. (1.7)). Conversely, if there exists a non-constant scalar $\theta$ satisfying (7.13) in which $i_{(1)}^{a}, i_{(2)}^{a}$ form an isometric net, then (7.17) are true. For, in this case, $\delta_{2} \alpha=\delta_{1} \beta$, so that (7.17) is a consequence of (7.14). The curvature conic $(G)$ of the $V_{2}$ in $S_{4}$ is the line segment:

$$
\begin{aligned}
& z=\rho_{1} \cos ^{2} \phi+\rho_{2} \sin ^{2} \phi, \\
& t=\sigma_{1} \cos ^{2} \phi+\sigma_{2} \sin ^{2} \phi \quad\left(\sigma_{1} \sigma_{2}=C^{\prime}-C=\text { const. }\right) .
\end{aligned}
$$

An example of such $V_{2}$ in $S_{3}$ is obtained by putting $\alpha=\beta=0$. Then $K=0$ and the fundamental equations (7.2) reduce to $C^{\prime}=-\rho_{1} \rho_{2}, \delta_{2} \rho_{1}=0, \delta_{1} \rho_{2}=0$, which are equivalent to

or to

$$
C^{\prime} \neq 0, \quad \rho_{1}=\text { const. } \neq 0, \quad \rho_{2}=-C^{\prime} / \rho_{1}=\text { const. }
$$

$$
C^{\prime}=0, \quad \rho_{1}=0, \quad \delta_{1} \rho_{2}=0 .
$$

The latter case gives a developable surface in an $R_{\mathbf{3}}$.

Finally, if none of the equations in (7.17) is satisfied, the last possible solution of $(7.14)$ is

$$
\epsilon e^{2 \theta}=\frac{\delta_{1} \beta-\alpha \beta}{\delta_{2} \alpha-\alpha \beta} \neq 1, \quad \text { that is, } \quad 2 \theta=\log \frac{\epsilon\left(\delta_{1} \beta-\alpha \beta\right)}{\left(\delta_{2} \alpha-\alpha \beta\right)} .
$$

Using this in (7.13) we have

$$
\begin{aligned}
& \delta_{1} \log \frac{\epsilon\left(\delta_{1} \beta-\alpha \beta\right)}{\left(\delta_{2} \alpha-\alpha \beta\right)}=2 \alpha \frac{\delta_{2} \alpha-\delta_{1} \beta}{\delta_{2} \alpha-\alpha \beta} \neq 0, \\
& \delta_{2} \log \frac{\epsilon\left(\delta_{1} \beta-\alpha \beta\right)}{\left(\delta_{2} \alpha-\alpha \beta\right)}=2 \beta \frac{\delta_{2} \alpha-\delta_{1} \beta}{\delta_{1} \beta-\alpha \beta} \neq 0 .
\end{aligned}
$$

If the lines of curvature of $V_{2}$ in $S_{3}$ satisfy these equations, the function $\theta$ and consequently $\sigma_{1}, \sigma_{2}$, are uniquely determined. Therefore, we have here-a uniquely determined desirable imbedding of the isometric $V_{2}$ in $S_{4}$. The curvature conic for this $V_{2}$ in $S_{4}$ is the line segment (7.18).

From (7.13) we can find the form of the $d s^{2}$, referred to the lines of curvature, for the $V_{2}$ of the cases (7.17) and (7.20). Take $i_{(1)}^{a}, i_{(2)}^{a}$ as parametric curves, then we have (1.12) and (1.13), and equations (7.13) become

$$
\frac{\partial_{u} \theta}{1-\epsilon e^{2 \theta}}=\partial_{u} \log G^{1 / 2}, \quad \frac{\partial_{v} \theta}{\epsilon e^{-2 \theta}-1}=\partial_{v} \log E^{1 / 2} .
$$

Integration of these gives

$$
E=\frac{U^{*}}{1-\epsilon e^{2 \theta}}, \quad G=\frac{V^{*} e^{2 \theta}}{1-\epsilon e^{2 \theta}},
$$


where $U^{*}, V^{*}$ are respectively some functions of $u, v$ alone. Therefore after an obvious transformation on $u$ and $v$, we have

$$
d s^{2}=\frac{\epsilon_{1} d u^{2}+\epsilon_{2} e^{2 \theta} d v^{2}}{1-\epsilon e^{2 \theta}}, \quad \quad \epsilon, \epsilon_{1}, \epsilon_{2}= \pm 1 .
$$

We have seen that the case (7.17) arises when $\theta \neq$ const. and $i_{(1)}^{a}, i_{(2)}^{a}$ form an isometric net, that is, when

$$
G / E=\epsilon_{1} \epsilon_{2} e^{2 \theta} \not \neq \text { const. }
$$

is the product of a function of $u$ with a function of $v$. Hence the linear element of this $V_{2}$ is

$$
d s^{2}=\frac{d u^{2} \pm d v^{2}}{U+V}
$$

where $U=U(u), V=V(v)$ are not both constant. We have thus completed the proof of the first part of the theorem.

Case 2. $C^{\prime}=C$. Equations (7.3) and (7.4) are equivalent to

$$
\sigma_{3}^{2}-\sigma_{1} \sigma_{2}=0, \quad \nu_{1} \sigma_{3}=\nu_{2} \sigma_{1} .
$$

If all the $\sigma$ 's are zero, it follows from (7.6) that

$$
\delta_{2} \nu_{1}-\delta_{1} \nu_{2}+\beta \nu_{1}-\alpha \nu_{2}=\dot{0} .
$$

In consequence of this, we can choose the normals $i_{(3)}^{\kappa}$, $i_{(4)}^{\kappa}$ to $V_{2}$ in $S_{4}$ so that $\nu_{1}=\nu_{2}=0$. For, from (7.24) and (1.4) it follows that a scalar $f$ exists so that

$$
\nu_{1}=\delta_{1} f, \quad \nu_{2}=\delta_{2} f, \quad \text { that is, } \quad v_{c}=\partial_{c} f \text {. }
$$

Now if we use

$$
i_{(3)}^{* k}=i_{(3)}^{*} \cos f+i_{(4)}^{*} \sin f, \quad i_{(4)}^{* k}=-i_{(3)}^{\kappa} \sin f+i_{(4)}^{k} \cos f
$$

as the normals to $V_{2}$ in $S_{4}$, the corresponding vector $v_{c}{ }^{*}$ is (cf. (2.3))

$$
v_{c}^{*}=B_{c}^{\lambda} i_{(3)}^{* x} \nabla_{\lambda} i_{k}^{*(4)}=v_{c}-B_{c}^{\lambda} \nabla_{\lambda} f=\partial_{c} f-\partial_{c} f=0,
$$

as was to be proved. This being the case, equations (7.3)-(7.6) are now all satisfied. But we have here only a trivial imbedding of an isometric $V_{2}$ in $S_{4}$, because the $V_{2}$ in $S_{3}$ may be considered as a $V_{2}$ in $S_{4}$. This proves (2a) of the theorem.

Let us suppose that not all the $\sigma$ 's are zero. Then we may choose $i_{(1)}^{a}$ so that

$$
\sigma_{3}=0 \text {. }
$$

Equation (7.23) now gives

$$
\sigma_{2}=0, \quad \sigma_{1} \neq 0, \quad \nu_{2}=0,
$$


and thence equations (7.5) and (7.6) become

$$
\delta_{2} \sigma_{1}+\beta \sigma_{1}+\nu_{1} \rho_{3}=0, \delta_{2} \nu_{1}+\beta \nu_{1}-\sigma_{1} \rho_{3}=0, \alpha \sigma_{1}+\nu_{1} \rho_{2}=0 .
$$

To study the consistency of these equations in $\sigma_{1}$ and $\nu_{1}$, we have to distinguish whether $\nu_{1}=0$ or $\nu_{1} \neq 0$.

If $\nu_{1}=0$, these equations reduce to

$$
\nu_{1}=0, \quad \alpha=0, \quad \rho_{3}=0, \quad \delta_{2} \sigma_{1}+\beta \sigma_{1}=0 .
$$

Equations $(7.28)_{2,3}$ show that the lines of curvature $i_{(2)}^{a}$ of $V_{2}$ in $S_{3}$ are geodesics in $V_{2}$. And it follows from (7.25), (7.26) and (7.28) $)_{1,4}$ that we have a desirable imbedding of an isometric $V_{2}$ in $S_{4}$ which depends on an arbitrary solution $\sigma_{1}$ of the linear partial differential equation (7.28) 4. This solution $\sigma_{1}$, when the linear element of $V_{2}$ is referred to $i_{(1)}^{a}, i_{(2)}^{a}$ as parametric curves $v=$ const., $u=$ const., is seen to depend on one arbitrary function of $u$. Hence the imbedding depends on an arbitrary function of a single variable. On account of (7.25) and (7.28) 3 , the curvature conic of $V_{2}$ in $S_{4}$ is a line segment. This proves (2c) of Theorem 7.1.

If $\nu_{1} \neq 0$, equations $(7.27)_{1,2}$ can be written

$$
\delta_{2} \log \sigma_{1}+\beta+\rho_{3} \frac{\nu_{1}}{\sigma_{1}}=0, \quad \delta_{2} \log \nu_{1}+\beta-\rho_{3} \frac{\sigma_{1}}{\nu_{1}}=0 .
$$

Subtraction gives $\delta_{2} \log \left(\sigma_{1} / \nu_{1}\right)+\left(\nu_{1} / \sigma_{1}+\sigma_{1} / \nu_{1}\right) \rho_{3}=0$, that is,

$$
\rho_{3}=-\frac{\delta_{2} \log \left(\sigma_{1} / \nu_{1}\right)}{\nu_{1} / \sigma_{1}+\sigma_{1} / \nu_{1}}=-\frac{\delta_{2} \sigma_{1} / \nu_{1}}{1+\left(\sigma_{1} / \nu_{1}\right)^{2}} .
$$

Therefore

$$
\rho_{3}=\delta_{2} \arctan \left(-\sigma_{1} / \nu_{1}\right) .
$$

At this stage we have to consider the cases $\alpha \neq 0$ or $\alpha=0$ separately.

If $\alpha \neq 0$, then it follows from $(7.26)_{2}$ and $(7.27)_{3}$ that $\rho_{2} \neq 0$. We eliminate $\sigma_{1} / \nu_{1}$ from (7.30) and $(7.27)_{3}$ and get

$$
\rho_{3}=\delta_{2} \arctan \left(\rho_{2} / \alpha\right) .
$$

This is a necessary condition for (7.27) to have a solution for $\sigma_{1}, \nu_{1}$. Evidently it is also sufficient. If (7.31) is satisfied, $\sigma_{1}$ is given by

$$
\delta_{2} \log \sigma_{1}+\beta-\rho_{3} \alpha / \rho_{2}=0,
$$

which is obtained from $(7.27)_{3}$ and (7.29) $)_{1}$ by elimination of $\nu_{1} / \sigma_{1}$. Therefore if $\sigma_{1}^{0}$ is a solution of (7.32), the most general solution will be

$$
\sigma_{1}=\mu \sigma_{1}^{0},
$$

where $\mu$ is any solution of $\delta_{2} \mu=0$. Hence referring $V_{2}$ to $i_{(1)}^{a}, i_{(2)}^{a}$ as parametric 
curves we see that for given $i_{(1)}^{a}$, $i_{(2)}^{a}$ satisfying (7.31), the solution for $\sigma_{1}, \nu_{1}$ depends on an arbitrary function of a single variable.

We now show that for any $V_{2}$ in $S_{3}$ there exist orthogonal congruences $i_{(1)}^{a}, i_{(2)}^{a}$ satisfying (7.31). Let $i_{(1)}^{a}, i_{(2)}^{a}$ be expressed in terms of two given orthogonal congruences $i_{(1)}^{* a}, i_{(2)}^{* a}$ :

$$
i_{(1)}^{a}=i_{(1)}^{* a} \cos \theta+i_{(2)}^{* a} \sin \theta, \quad i_{(2)}^{a}=-i_{(1)}^{* a} \sin \theta+i_{(2)}^{* a} \cos \theta .
$$

Then $\alpha=-\left(\delta_{1}^{*} \theta-\beta^{*}\right) \sin \theta+\left(\delta_{2}^{*} \theta+\alpha^{*}\right) \cos \theta$ and $\alpha$ is not identically zero. Consequently equation (7.31) will become a linear differential equation of the second order in $\theta$.

Hence we have here a solution of the problem in which the imbedding of the isometric $V_{2}$ in $S_{4}$ depends on an arbitrary function of a single variable and on an arbitrary solution of a linear partial differential equation of the second order in two independent variables. The curvature conic $(G)$ of such $V_{2}$ in $S_{4}$ is

$$
z=\rho_{1} \cos ^{2} \phi+\rho_{2} \sin ^{2} \phi+2 \rho_{3} \cos \phi \sin \phi, \quad t=\sigma_{1} \cos ^{2} \phi,
$$

which is in general nondegenerate. This proves (2b) of Theorem 7.1.

Let us now return to the stage where (7.30) appeared. If $\alpha=0$, then since $\nu_{1} \neq 0$ in the case under consideration, it follows from $(7.27)_{3}$ that $\rho_{2}=0$. Then the second fundamental tensor of $V_{2}$ in $S_{3}$ is

$$
h_{c b}=\rho_{1} i_{c}^{(1)} i_{b}^{(1)}+\rho_{3}\left(i_{c}^{(1)} i_{b}^{(2)}+i_{c}^{(2)} i_{b}^{(1)}\right),
$$

showing that $i_{(2)}^{a}$ is an asymptotic congruence of $V_{2}$ in $S_{3}$. Since $\alpha=0$ is the geodesic curvature of $i_{(2)}^{a}, V_{2}$ is generated by a one-parameter family of geodesics in $S_{3}$, that is, $V_{2}$ is a ruled surface in $S_{3}$.

Consider equation (7.30). Let $\theta^{0}$ be a solution of $\delta_{2} \theta=\rho_{3}$, then the most general solution of (7.30) is

$$
\arctan \left(-\sigma_{1} / \nu_{1}\right)=\theta^{0}+\mu,
$$

where $\mu$ is an arbitrary solution of $\delta_{2} \mu=0$. Therefore

$$
\sigma_{1} / \mu_{1}=-\tan \left(\theta^{0}+\mu\right) \text {. }
$$

Using this in (7.29) ${ }_{1}$ we get

$$
\delta_{2} \log \sigma_{1}+\beta-\rho_{3} \cot \left(\theta^{0}+\mu\right)=0 .
$$

If $\sigma_{1}^{0}$ is a solution of this equation, the most general solution is

$$
\sigma_{1}=\lambda \sigma_{1}^{\mathrm{c}}
$$

where $\lambda$ is an arbitrary solution of $\delta_{2} \lambda=0$. Hence in this case we have a solution for $\sigma_{1}, \nu_{1}$ depending on two arbitrary solutions of the linear differential equation $\delta_{2} \lambda=0$, that is, we have a desirable imbedding of an isometric $V_{2}$ 
in $S_{4}$ which depends on two arbitrary functions of a single variable. The curvature conic $(G)$ of the isometric $V_{2}$ in $S_{4}$ is

$$
z=\rho_{1} \cos ^{2} \phi+2 \rho_{3} \sin \phi \cos \phi, \quad t=\sigma_{1} \cos ^{2} \phi,
$$

which is in general nondegenerate. Since $\sigma_{2}=\rho_{2}=0$, the isometric $V_{2}$ in $S_{4}$ is also a ruled surface in $S_{4}$. This proves the last part of Theorem 7.1.

\section{REFERENCES}

1. M. Bocher, Introduction to higher algebra, New York, 1908.

2. O. Borůvka, Sur une classe de surfaces minima plonges dans un espace d quatre dimensions d courboure constante, Bulletin international Česká akademie véd a umęní v Praze vol. 29 (1928) pp. 256-277.

3. R. Calapso, Sulle reti di Voss di uno spazio lineare quadri dimensionale, Rendiconti Seminario matematico Roma (4) vol. 2 (1938) pp. 276-311.

4. - Sulle deformazione delle reti di Voss di un $S_{4}$ euclideo, Atti Accademia nazionale dei Lincei (4) vol. 28 (1939) pp. 231-236.

5. N. Coburn, Surfaces in four-space of constant curvature, Duke Math. J. vol. 5 (1939) pp. 30-38.

6. L. P. Eisenhart, Minimal surfaces in Euclidean four space, Amer. J. Math. (4) vol. 34 (1912) pp. 215-236.

7. - Differential geometry, New York, 1909.

8. - Riemannian geometry, Princeton, 1926.

9. W. Graustein, Invariant methods in classical differential geometry, Bull. Amer. Math. Soc. vol. 36 (1930) pp. 489-521.

10. J. Knoblauch, Grundlagen der Differentialgeometrie, Leipzig, 1913.

11. K. Kommerell, Riemannsche Flächen im ebenen Raum von vier Dimensionen, Math. Ann. vol. 60 (1905) pp. 546-596.

12. S. Kwietniewski, Über Flächen des vierdimensionalen Raumes, deren sämtliche Tangentialebenen untereinander gleichwinklig sind, und ihre Beziehung zu den ebenen Kurven, Dissertation, Zürich.

13. C. L. E. Moore, and E. B. Wilson, Differential geometry of two-dimensional surfaces in hyperspace, Proceedings of the American Academy of Arts and Sciences vol. 52 (1916) pp. 267368.

14. G. Ricci, Lezioni sulla teoria della superficie, Verona and Padova, Druker, 1898.

15. J. A. Schouten, and D. J. Struik, Einfithrung in die neueren Methoden der Differentialgeometrie II, Batavia, 1938.

16. C. Tompkins, Isometric imbedding of flat manifolds in Euclidean space, Duke Math. J. vol. 5 (1939) pp. 58-61.

17. C. Zitto, Reti di Voss a curvatura nulla di un $S_{\mathbf{1}}$ euclideo, Atti Acad. Pelororitana vol. 41 (1939) pp. 44-47.

University of Pennsylvania, Philadelphia, Pa. 